\title{
On the projective geometry of rational homogeneous varieties
}

\author{
Joseph M. Landsberg and Laurent Manivel
}

\begin{abstract}
We determine the varieties of linear spaces on rational homogeneous varieties, provide explicit geometric models for these spaces, and establish basic facts about the local differential geometry of rational homogeneous varieties.
\end{abstract}

Mathematics Subject Classification (2000). 14M15, $20 \mathrm{G} 05$.

Keywords. Flag variety, fundamental forms, linear spaces, octonion.

\section{Introduction}

Let $G$ be a complex simple Lie group and $P_{S}$ a parabolic subgroup corresponding to a subset $S$ of nodes of the Dynkin diagram (so that a maximal parabolic subgroup is defined by a single root). Then $G / P_{S}$ has a minimal homogeneous embedding in the projective space of the highest weight module of $G$ corresponding to the weight $\lambda=\sum_{i \in S} \omega_{i}$, where $\omega_{i}$ is the $i$-th fundamental weight. We study the local differential geometry of the embedded variety $G / P_{S} \subset \mathbb{P} V_{\lambda}$ and the projective linear subspaces on $G / P_{S} \subset \mathbb{P} V_{\lambda}$.

We describe the varieties parametrizing such linear spaces in $\S 4-6$. In most cases (those of "non-short roots") the parameter varieties are determined in terms of Dynkin diagram data as explained in §4. (See in particular Theorem 4.9.) The exceptional (exposed short root) cases are determined by use of explicit models in $\S 5$ for the case of classical groups and $\S 6$ for the exceptional groups. In all cases, each connected component of the variety of linear spaces on a $G / P$ is quasihomogeneous; more precisely, it is the union of a finite number of $G$-orbits.

The case of unirulings by lines was studied in [4] by means of Tits buildings. Our approach is by means of projective differential geometry. This method is well suited because the variety $G / P_{S}$ is homogeneous and in particular cut out by quadrics, so the varieties of linear spaces on it are determined by second order data at a point $x \in G / P_{S}$.

In $\S 2$ we establish basic connections between local differential geometry and representation theory. We study the semisimple part $H$ of $P_{S}$, which fixes the point 
$x=\left[v_{\lambda}\right]$ corresponding to the highest weight line in its action on the tangent space $T_{x} G / P_{S}$. As an $H$-module, $T_{x} G / P_{S}$ decomposes into a direct sum of generalized minuscule $H$-modules. If $S=\{\alpha\}$ where $\alpha$ is a non-short root, the space of tangent directions to lines is a minuscule variety of $H$ (an irreducible, minimally homogeneously embedded Hermitian symmetric space of $H$ ).

We study minuscule varieties in $\S 3$ and prove our main result on their infinitesimal geometry in $\S 4$.

This is the first paper in a series $[15,14,16,17,18]$ establishing new relations between the representation theory of complex simple Lie groups and the algebraic and differential geometry of their homogeneous varieties. The surprising connection between secant varieties and prolongations developed in this paper is exploited in the sequels.

Acknowledgements. We thank J. Wolf, J.-M. Hwang and D. Snow for useful conversations, and an anonymous referee for the simplified proof of Theorem 4.3.

\section{Under the microscope}

In this section we establish the basic connections between differential invariants of homogeneous varieties and representation theoretic data. In $\S 2.1$ we review the projective fundamental forms of an arbitrary projective variety $X^{n} \subset \mathbb{P}^{n+a}$ and establish a connection between secant varieties and fundamental forms. In $\S 2.2$ we express the fundamental forms of homogeneously embedded homogeneous varieties $X=G / P \subset \mathbb{P} V_{\lambda}$ in terms of the universal envelopping algebra $U(\mathfrak{g})$. In $\S 2.3$ we discuss the $P$-module structure on $T_{x} X$, introduce an important class of homogeneous varieties, the generalized minuscule varieties and explain their role in the study of fundamental forms of rational homogeneous varieties.

\subsection{Fundamental forms of projective varieties}

\subsubsection{Notation}

We let $V=\mathbb{C}^{n+a+1}$ and $\mathbb{P} V$ the corresponding projective space. If $Y \subset \mathbb{P} V$ is a set, we let $\hat{Y} \subset V$ denote the corresponding cone in $V$. If $v \in V$, we let $[v] \in \mathbb{P} V$ denote the corresponding point in projective space. For any vector space $W$, we let $W^{*}$ denote the dual vector space. Let $X \subset \mathbb{P} V=\mathbb{P}^{n+a}$ be a projective variety of dimension $n$, and let $x \in X$ be a smooth point. We let $T_{x} X$ denote the (intrinsic) Zariski tangent space to $X$ at $x, \tilde{T}_{x} X \subset \mathbb{P}^{n+a}$ denote the embedded tangent projective space (the $\mathbb{P}^{n} \subset \mathbb{P}^{n+a}$ that best approximates $X$ at $x$ ), and $\hat{T}_{x} X=\widehat{\tilde{T}_{x} X} \subset V$. We have the relation $T_{x} X=\hat{x}^{*} \otimes\left(\hat{T}_{x} X\right) / \hat{x}$ and we also have, for any $p \in V$ with $x=[p], \hat{T}_{x} X=T_{p}^{\text {affine }} \hat{X}$, the affine tangent space at $p$.

We let $N_{x} X=T_{x} \mathbb{P} V / T_{x} X$ denote the normal space of $X$ at $x$. 


\subsubsection{Fundamental forms in coordinates}

Let $X^{n} \subset \mathbb{P}^{n+a}$ be a projective variety, and let $x \in X$ be a smooth point. Take local linear coordinates $\left(x^{1}, \ldots, x^{n}, x^{n+1}, \ldots, x^{n+a}\right)$ adapted to $x$, which means that they are centered at $x$ and that $x^{n+1}=\cdots=x^{n+a}=0$ are equations of the embedded tangent space of $X$ at $x$. Write $X$, locally in the complex topology, as a graph

$$
x^{\mu}=\sum_{1 \leq \alpha, \beta \leq n} q_{\alpha \beta}^{\mu} x^{\alpha} x^{\beta}+\sum_{1 \leq \alpha, \beta, \gamma \leq n} r_{\alpha \beta \gamma}^{\mu} x^{\alpha} x^{\beta} x^{\gamma}+\ldots,
$$

where $n+1 \leq \mu \leq n+a$. The geometric information in the series (that is, information independent of choice of adapted coordinates) can be encoded in a series of tensors, the simplest of which is the projective second fundamental form

$$
\mathbb{F F}_{X, x}^{2}=\sum_{\substack{1 \leq \alpha, \beta \leq n, n+1 \leq \mu \leq n+a}} q_{\alpha \beta}^{\mu} d x^{\alpha} \circ d x^{\beta} \otimes \frac{\partial}{\partial x^{\mu}} \in S^{2} T_{x}^{*} X \otimes N_{x} X .
$$

If $x$ is a general point, $\mathbb{F F}_{X, x}^{2}$ even contains information about the global geometry of $X$, see [9], [12]. It is useful to consider the second fundamental form as a system of quadrics $\left|\mathbb{F}_{X, x}^{2}\right|:=\mathbb{P}\left(\mathbb{F F}_{X, x}^{2}\left(N_{x}^{*} X\right)\right) \subseteq \mathbb{P} S^{2} T_{x}^{*} X$ parametrized by $N_{x}^{*} X$, and Base $\left|\mathbb{F F}_{X, x}^{2}\right| \subset \mathbb{P} T_{x} X$, their common zero locus.

We let $\hat{T}_{x}^{(2)} X=\hat{T}_{x} X+\mathcal{O}(1)_{x} \otimes \mathbb{F F}_{X, x}^{2}\left(N_{x}^{*} X\right) \subset V$, the second osculating space to $X$ at $x$, and $N_{2}=N_{2, x} X=\mathcal{O}(-1) \otimes\left(\hat{T}_{x}{ }^{(2)} X / \hat{T}_{x} X\right)$.

More generally, the $k$-th projective fundamental form of $X$ at $x$ is a tensor

$$
\mathbb{F F}_{X, x}^{k} \in S^{k} T_{x}^{*} X \otimes N_{k, x} X
$$

where $N_{k}=N_{k, x} X=\mathcal{O}(-1)_{x} \otimes\left(\hat{T}_{x}{ }^{(k)} X / \hat{T}_{x}{ }^{(k-1)} X\right)$ and $\hat{T}_{x}{ }^{(k)} X=\hat{T}_{x}{ }^{(k-1)} X+$ $\mathcal{O}(-1)_{x} \otimes \mathbb{F}^{k}\left(S^{k} T_{x} X\right)$ is the $k$-th osculating space to $X$ at $x$. To define $\mathbb{F}^{k}{ }_{X, x}$ one can use the same definitions as one does for the Euclidean fundamental forms, either in coordinates or as the derivatives of successive Gauss mappings (see [12]). Note that the osculating spaces determine a flag of $V$,

$$
0 \subset \hat{x} \subset \hat{T}_{x} X \subset \hat{T}_{x}^{(2)} X \subset \ldots \subset \hat{T}_{x}^{(f)}=V .
$$

More generally, given a mapping $\phi: Y \rightarrow \mathbb{P} V$, one defines its fundamental forms $\mathbb{F F}_{\phi}^{k}$ in the same manner. $\mathbb{F F}_{\phi, x}^{2}$ quotiented by ker $\phi_{* x}$ is isomorphic to the second fundamental form of the image, $\mathbb{F}_{\phi(Y), \phi(x)}^{2}$. See [13] for details.

In what follows, we slightly abuse notation by ignoring twists by the line bundles $\mathcal{O}(j)$, which will not matter as we study fundamental forms only at some fixed base point. We let $\left|\mathbb{F}_{X, x}^{k}\right| \subset \mathbb{P} S^{k} T_{x}^{*} X$ denote $\mathbb{P}\left(\mathbb{F F}_{X, x}^{k}\left(N_{k, x}^{*} X\right)\right)$ and Base $\left|\mathbb{F}_{X, x}^{k}\right| \subset$ $\mathbb{P} T_{x} X$ denote its base locus.

\subsubsection{Prolongation}

Let $V$ be a vector space, let $A \subset S^{d} V^{*}$ be a linear subspace, and let

$$
A^{(l)}:=\left(A \otimes S^{l} V^{*}\right) \cap S^{d+l} V^{*},
$$


the -th prolongation of $A$. Here the inclusion $S^{d+l} V^{*} \hookrightarrow S^{d} V^{*} \otimes S^{l} V^{*}$ is dual to the multiplication map $S^{d} V \otimes S^{l} V \longrightarrow S^{d+l} V$. If $P \in S^{d+l} V^{*}$, we still denote by $P\left(v_{1}, \ldots, v_{d+l}\right)$ its polarization. Then $P \in A^{(l)}$ if and only if for all $v \in V$, the degree $d$ polynomial $w \mapsto P(v, \ldots, v, w, \ldots, w)$ belongs to $A$. The notation is such that $A^{(0)}=A$.

Let $\operatorname{Jac}(A):=\{v\lrcorner P \mid v \in V, P \in A\} \subseteq S^{d-1} V^{*}$, the Jacobian space of $A$. Then $A^{(1)}=\left\{P \in S^{d+1} V^{*} \mid \operatorname{Jac}(P) \subset A\right\}$. Here $\lrcorner$ denotes contraction, $\left.v\right\lrcorner P\left(w_{1}, \ldots, w_{d-1}\right)$ $=P\left(v, w_{1}, \ldots, w_{d-1}\right)$.

A basic fact about fundamental forms, due to Cartan ([2], p. 377) (and rediscovered in [9]), is that if $x \in X$ is a general point, then the prolongation property holds at $x$ :

$$
\left|\mathbb{F} \mathbb{F}_{X, x}^{k}\right| \subseteq\left|\mathbb{F}_{X, x}^{k-1}\right|^{(1)}
$$

A geometric consequence is as follows. Define the $k$-th secant variety $\sigma_{k}(Y)$ of a projective variety $Y \subset \mathbb{P}^{N}$ to be the closure of the union of the linear spaces spanned by $k$ points of $Y$. The notation is such that $\sigma_{1}(Y)=Y$.

Proposition 2.1. Let $X^{n} \subset \mathbb{P}^{n+a}$ be a variety and $x \in X$ a general point. Then for $k \geq 2$,

$$
\text { Base }\left|\mathbb{F F}_{X, x}^{k}\right| \supseteq \sigma_{k-1}\left(\text { Base }\left|\mathbb{F F}_{X, x}^{2}\right|\right) \text {. }
$$

Proposition 2.1 is a consequence of the following lemma:

Lemma 2.2. Let $A \subset S^{2} V^{*}$ be a system of quadrics with base locus Base $(A) \subset$ $\mathbb{P} V$. Then

$$
\operatorname{Base}\left(A^{(k-1)}\right) \supseteq \sigma_{k}(\operatorname{Base}(A)) .
$$

Moreover, if Base $(A)$ is linearly non-degenerate, then for $k \geq 2, I_{k}\left(\sigma_{k}(\operatorname{Base}(A))=\right.$ 0 , and if $A=I_{2}($ Base $(A))$, then $I_{k+1}\left(\sigma_{k}\right.$ (Base $\left.(A)\right)=A^{(k)}$, where $I_{d}(Z) \subset S^{d} V^{*}$ is the component of the ideal of $Z \subset \mathbb{P} V$ in degree $d$.

Proof. We prove the lemma for $k=2$, the generalization being clear. We first need to prove that any polynomial $P \in A^{(1)}$ vanishes on $v=s x+t y$ for all $s, t \in \mathbb{C}$ and $x, y \in B$, the cone over Base $(A)$. Since $P(x, x, \cdot)=P(y, y, \cdot)=0$, we have

$P(v)=P(v, v, v)=s^{3} P(x, x, x)+3 s^{2} t P(x, x, y)+3 s t^{2} P(x, y, y)+t^{3} P(y, y, y)=0$.

Now, say $Q \in I_{2}\left(\sigma_{2}(\operatorname{Base}(A))\right.$. Then for all $x, y \in B$ and $s, t \in \mathbb{C}, Q(s x+t y)=$ 0 , which implies $Q(x, y)=0$, which implies $Q=0$ since Base $(A)$ is non-degenerate.

Finally, consider a polynomial $P \in I_{3}\left(\sigma_{2}(B(A))\right.$. Since $P$ vanishes on $v=$ $s x+t y$ for all $x, y \in B$ and all $s, t \in \mathbb{C}$, we have $P(x, x, y)=0$ for all $x \in B$, and all $y \in B$, hence all $y \in V$ since $\operatorname{Base}(A)$ is non-degenerate. Thus for all $y \in V$, $P(\cdot, \cdot, y)$ is a quadric vanishing on $\operatorname{Base}(A)$, hence belongs to $A=I_{2}(\operatorname{Base}(A))$. This means that $P$ is in $A^{(1)}$.

An elementary fact about projective varieties is that if $X^{n} \subset \mathbb{P}^{n+a}$ is a variety whose ideal is generated in degree $\leq d$, and $L$ a linear space osculating to order 
$d$ at a smooth point $x \in X$, then $L \subset X$. The ideal of a projective homogeneous variety is generated in degree two (see e.g. [19]), so if $X \subset \mathbb{P} V$ is homogeneous, then Base $\left|\mathbb{F F}_{X, x}^{2}\right|$ is the set of tangent directions to lines on $X$ through $x$. If $y \in \tilde{T}_{x} X \cap X$ then the line $\mathbb{P}_{x y}^{1}$ is contained in $X$.

\subsection{Osculating spaces of homogeneous varieties}

Let $G$ be a simply connected complex semi-simple Lie group, $\mathfrak{g}$ its Lie algebra, $\mathfrak{g}^{\otimes}$ the tensor algebra of $\mathfrak{g}$ and $U(\mathfrak{g})=\mathfrak{g}^{\otimes} /\{x \otimes y-y \otimes x-[x, y] \mid x, y \in \mathfrak{g}\}$, the universal envelopping algebra. $U(\mathfrak{g})$ inherits a filtration from the natural grading of $\mathfrak{g}^{\otimes}$, and the associated graded algebra is the symmetric algebra of $\mathfrak{g}$. Fix a maximal torus $T$ and a Borel subgroup $B$ of $G$ containing $T$. We adopt the convention that $B$ is generated by the positive roots, and we write the corresponding root space decomposition of $\mathfrak{g}$ as

$$
\mathfrak{g}=\mathfrak{t} \oplus \bigoplus_{\alpha \in \Delta_{+}}\left(\mathfrak{g}_{\alpha} \oplus \mathfrak{g}_{-\alpha}\right),
$$

where $\Delta_{+}$denotes the set of positive roots. We use the ordering of the roots as in [1].

Let $V_{\lambda}$ be an irreducible $G$-module with highest weight $\lambda$, and $v_{\lambda} \in V_{\lambda}$ a highest weight vector. The induced action of $\mathfrak{g}$ extends to the universal envelopping algebra, inducing a filtration of $V_{\lambda}$ whose $k$-th term is

$$
V_{\lambda}^{(k)}=U_{k}(\mathfrak{g}) v_{\lambda} .
$$

Let $x=\left[v_{\lambda}\right] \in \mathbb{P} V_{\lambda}$ and let $X=G / P \subset \mathbb{P} V_{\lambda}$ be its $G$-orbit. Here $P$ is the stabilizer of $x$, it is a parabolic subgroup of $G$. The tangent bundle $T X$ is a homogeneous bundle and we identify $T_{x} X$ with the associated $P$-module $\mathfrak{g} / \mathfrak{p}$. The osculating spaces and the fundamental forms of $X$ have a simple representationtheoretic interpretation:

Proposition 2.3. Let $X=G / P \subset \mathbb{P} V_{\lambda}$ be a homogeneous variety with base point $x=\left[v_{\lambda}\right]$. Let $\hat{T}_{x}^{(k)} X$ denote the cone over the $k$-th osculating space at $x$ and let $N_{k}=\hat{T}_{x}^{(k)} X / \hat{T}_{x}^{(k-1)} X$ be the $k$-th normal space twisted by $\mathcal{O}(-1)$. Then

$$
\hat{T}_{x}^{(k)} X=V_{\lambda}^{(k)}, \quad N_{k}=V_{\lambda}^{(k)} / V_{\lambda}^{(k-1)} .
$$

Moreover, there is a commutative diagram

$$
\begin{array}{rcc}
S^{k} \mathfrak{g} & = & U_{k}(\mathfrak{g}) / U_{k-1}(\mathfrak{g}) \\
\downarrow & & \downarrow \\
\mathbb{F} \mathbb{F}_{X, x}^{k}: S^{k} T_{x} X & \longrightarrow & N_{k},
\end{array}
$$

where the bottom horizontal map is the $k$-th fundamental form at $x$.

Proof. The diagram above is the $k$-th fundamental form of the mapping $\phi: G \rightarrow$ $\mathbb{P} V$ at $e \in G$, where $\phi(G)=X$. 
$V_{\lambda}^{(k)}$ has a natural $P$-module structure. Thus the osculating spaces of $X$ at $x$ correspond to the increasing filtration of $P$-modules

$$
0 \subset \hat{x} \subset \hat{T}_{x} X=V_{\lambda}^{(1)} \subset V_{\lambda}^{(2)} \subset \cdots \subset V_{\lambda}^{(f)}=V_{\lambda} .
$$

Our next goal is to understand the first quotient of this filtration, namely the structure of $T_{x} X$ as a $P$-module.

\subsection{Decomposing the tangent space}

Let $P=P_{\alpha_{i}}$ denote the maximal parabolic subgroup of $G$ corresponding to the simple root $\alpha_{i}$. Let $P=L P^{u}$ be a Levi decomposition of $P$, where $P^{u}$ is unipotent, $L$ is reductive and contains the maximal torus $T$. If $\alpha$ is a positive root, let $\alpha=\sum_{j} m_{j}(\alpha) \alpha_{j}$ be its decomposition in terms of simple roots. Let $\Delta_{X}=\{\alpha \in$ $\left.\Delta_{+} \mid m_{i}(\alpha)>0\right\}$. We have the root space decompositions

$$
\begin{aligned}
\mathfrak{p} & =\mathfrak{t} \oplus\left(\bigoplus_{\alpha \in \Delta_{+}} \mathfrak{g}_{\alpha}\right) \oplus\left(\bigoplus_{\alpha \in \Delta_{+} \backslash \Delta_{X}} \mathfrak{g}_{-\alpha}\right), \\
\mathfrak{l} & =\mathfrak{t} \oplus \bigoplus_{\alpha \in \Delta_{+} \backslash \Delta_{X}}\left(\mathfrak{g}_{\alpha} \oplus \mathfrak{g}_{-\alpha}\right), \\
\mathfrak{p}^{u} & =\bigoplus_{\alpha \in \Delta_{X}} \mathfrak{g}_{\alpha} .
\end{aligned}
$$

Proposition 2.4. Let $G$ be simple, let $\tilde{\alpha}$ be the highest root of $\mathfrak{g}$, let $\alpha_{i}$ be a simple positive root, and let $P=P_{\alpha_{i}}$ be the associated maximal parabolic subgroup. For $1 \leq k \leq m_{i}(\tilde{\alpha})$, let

$$
\mathfrak{s}_{k}=\bigoplus_{m_{i}(\alpha) \geq-k} \mathfrak{g}_{\alpha} .
$$

This defines an increasing filtration of $\mathfrak{g} / \mathfrak{p}$ by P-submodules. The quotients

$$
T_{k}=\bigoplus_{m_{i}(\alpha)=k} \mathfrak{g}_{-\alpha}
$$

are irreducible P-modules.

Proof. The fact that each $\mathfrak{s}_{k} / \mathfrak{s}_{k-1}$ is a $P$-module is clear. The irreducibility of $T_{k}$ is a special case of [25], 8.13.3 (which is attributed to Kostant).

The irreducibility of $T_{k}$ implies that the set $\left\{\alpha \in \Delta_{+} \mid m_{i}(\alpha)=k\right\}$ has a unique minimal element which we denote by $-\phi_{k}$ when we consider the root as a weight of $T_{k}$. In particular, the highest weight of $T_{1}$ is

$$
\phi_{1}=-\alpha_{i}=-\sum_{j} n\left(\alpha_{i}, \alpha_{j}\right) \omega_{j}
$$

where $n\left(\alpha_{i}, \alpha_{j}\right)$ denotes the entries of the Cartan matrix. This weight is easy to read directly on the Dynkin diagram of $G$. Let $H$ denote the semi-simple part of $L$. As an $H$-module, the filtration of $T_{x} X$ into irreducible $P$-modules becomes 
a direct sum decomposition into irreducible $H$-modules. Note that $L$ has a one dimensional center and that the Lie algebra of $H$ is

$$
\mathfrak{h}=\operatorname{ker} \omega_{i} \oplus \bigoplus_{\alpha \in \Delta_{+} \backslash \Delta_{X}}\left(\mathfrak{g}_{\alpha} \oplus \mathfrak{g}_{-\alpha}\right),
$$

where $\operatorname{ker} \omega_{i} \subset \mathfrak{t}$. The Dynkin diagram of $H$ is therefore deduced from that of $G$ by suppressing the node corresponding to the simple root $\alpha_{i}$. In particular, we conclude:

Proposition 2.5. Let $X=G / P$ be a homogeneous variety with $P$ a maximal parabolic and let $H$ be the semi-simple part of $P$. Then $T_{1}$, the first irreducible component of $T_{x} X$ as an $H$-module, is obtained by marking the nodes of $\mathcal{D}(H)$ adjacent to the node from $\mathcal{D}(G)$ that was removed. A node $\beta$ is given multiplicity two (resp. three) if there is an arrow emanating from $\alpha$ towards $\beta$ with a double (resp. triple) bond.

The above observations can be found in [7].

Definition 2.6. A fundamental weight $\omega_{i}$ is minuscule if the Weyl group acts transitively on the set of weights of the corresponding fundamental representation.

In an irreducible root system, a fundamental weight $\omega_{i}$ is cominuscule if the highest root has coefficient one on $\alpha_{i}$. In a reducible root system, a weight is cominuscule if it is a sum of cominuscule fundamental weights, one for each irreducible factor of the root system.

The relation between these two notions is as follows. In the irreducible case, $\omega_{i}$ is minuscule if and only if in the dual root system, the highest root has coefficient one on the coroot $\check{\alpha}_{i}([1]$, Chap. 8).

Geometrically, when $G$ is simple, the weight $\omega_{i}$ is cominuscule exactly when $G / P_{i}$ admits the structure of an irreducible Hermitian symmetric space whose automorphism group is locally isomorphic to $G$ (we call $G / P_{i}$ a $G$-Hermitian symmetric space). This was pointed out by Kostant in [11]. We use the following definition (be careful that minuscule varieties are in correspondence with cominuscule weights, not minuscule weights!):

Definition 2.7. A $G$-minuscule variety $X=G / P \subset \mathbb{P} V$ is a $G$-Hermitian (not necessarily irreducible) symmetric space in its minimal homogeneous embedding. A generalized minuscule variety is a Hermitian symmetric space $X=G / P \subset \mathbb{P} V$ in some $G$-homogeneous embedding, but the automorphism group of $X$ need not be locally isomorphic to $G$, and the embedding need not be minimal.

Proposition 2.8. Let $X=G / P$ with $G$ simple, and $P=P_{\alpha_{i}}$ a maximal subgroup with semi-simple part $H$. If $\alpha_{i}$ is not short, then the closed $H$-orbit $Y_{1} \subset \mathbb{P} T_{1}$ is an $H$-minuscule variety. 
See 2.11 below for a more general statement.

Proof. The observations above imply that $Y_{1}=H_{1} / Q_{1} \times \cdots \times H_{r} / Q_{r}$, where $H_{1}, \ldots, H_{r}$ are simple Lie groups whose Dynkin diagrams are the branches from $\alpha_{i}$ of $\mathcal{D}(G)$, and $Q_{1}, \ldots, Q_{r}$ are maximal parabolic subgroups defined for each branch by the node adjacent to $\alpha_{i}$. Since the root system of each $H_{k}$ is formed by the roots of $G$ with support on the corresponding branch, we just need to prove that if a root $\beta$ of $G$ has coefficient $m_{i}(\beta)=0$ on $\alpha_{i}$, its coefficient on an adjacent root $\alpha_{k}$ cannot exceed one. But this follows immediately from the equality $n\left(\beta, \alpha_{i}\right)=\sum_{j} m_{j}(\beta) n\left(\alpha_{j}, \alpha_{i}\right)$ (where the integers $n\left(\alpha_{j}, \alpha_{i}\right)$ are non-positive, and negative exactly when $\alpha_{j}$ is adjacent to $\alpha_{i}$ ), and the fact that, since $\alpha_{i}$ is not short, $\left|n\left(\beta, \alpha_{i}\right)\right| \leq 1$. The minimality of the embedding of $Y_{1}$ in $\mathbb{P} T_{1}$ similarly follows from the fact that $n\left(\alpha_{j}, \alpha_{i}\right)=-1$ for $\alpha_{j}$ connected to $\alpha_{i}$.

We can say slightly more when $G$ is simply laced.

Proposition 2.9. Let $G$ be a simple Lie group of type $A, D$ or $E$, let $P$ be a maximal parabolic subgroup, let $T_{1} \subset T_{x} G / P$ be as in 2.4. Then $T_{1}$ is a minuscule $H$-module.

Proof. The weights of $T_{1}$ are, by definition, the opposites of the roots $\beta$ such that $m_{i}(\beta)=1$. If $G$ is simply laced, these roots all have the same length and lemma 4.4 shows that the Weyl group of $H$ acts transitively on them.

The above discussion can easily be extended to homogeneous spaces $X=G / P$ with $P$ not necessarily maximal. Suppose that $P=P_{S}$ is the parabolic subgroup generated by the complement of a set $S$ of simple positive roots. Then there is an irreducible component of the $L$-module $T=T_{x}(G / P)$ for each choice of the coefficients of the positive roots on these simple roots. If we choose such a family of coefficients $a=\left(a_{i}\right)_{i \in S}$, and let

$$
\mathfrak{s}_{a}=\bigoplus_{\alpha \in \Delta_{+}, m_{i}(\alpha) \geq-a_{i}} \mathfrak{g}_{\alpha} \text { and } T_{a}=\bigoplus_{\alpha \in \Delta_{+}, m_{i}(\alpha)=a_{i}} \mathfrak{g}_{-\alpha}
$$

then $\mathfrak{s}_{a}$ is a $P$-submodule of $\mathfrak{g} / \mathfrak{p}$, and $T_{a}$ is an irreducible $L$-submodule of $\mathfrak{s}_{a}$. An important difference with the case of maximal parabolics is that the incidence relations between the non-zero $\mathfrak{s}_{a}$ 's is no longer a simple chain of inclusion, but defines a partial order.

Let $\varepsilon_{i}$ be the family of coefficients $a_{i}=1$, and $a_{j}=0$ for $j \in S-i$. The analogues of $T_{1}$ in the maximal case are the $H$-modules $T_{\varepsilon_{i}}$ (note that only the irreducible factors of $H$ corresponding to the branches of $\mathcal{D} \backslash S$ connected to $\alpha_{i}$ act non trivially on $T_{\varepsilon_{i}}$; we denote their product by $H_{\varepsilon_{i}}$ ). Again we need to know whether $\alpha_{i}$ is short or not, but this condition is relevant only with respect to a subdiagram of $\mathcal{D}(G)$. 
Definition 2.10. We call $\alpha \in S$ an exposed short root if the connected component of $\alpha$ in $\mathcal{D} \backslash(S \backslash \alpha)$ contains a root longer than $\alpha$, i.e., if an arrow in $\mathcal{D} \backslash(S \backslash \alpha)$ points towards $\alpha$.

Proposition 2.11. Notations as above. Let $Y_{1}^{i} \subset \mathbb{P} T_{\varepsilon_{i}}$ be the closed orbit. Then $Y_{1}^{i}$ is a generalized minuscule variety. Moreover, it is a $H_{\varepsilon_{i}}$-minuscule variety, except for situations equivalent to the following cases:

1. $C_{n} / P_{k}$ for $k<n$. Here $Y_{1}=S e g\left(\mathbb{P}^{k-1} \times \mathbb{P}^{2 n-2 k-1}\right), H=S L_{k} \times S p_{2 n-2 k} \subsetneq$ $S L_{k} \times S L_{2 n-2 k}$.

2. $C_{n} / P_{n}$. Here $Y_{1}=v_{2}\left(\mathbb{P}^{n-1}\right)$, which is $A_{n-1}$-minuscule but not in its minimal embedding.

3. $F_{4} / P_{4}$. Here $Y_{1}=B_{3} / P_{3}$, a six-dimensional quadric.

4. $G_{2} / P_{2}$. Here $Y_{1}=v_{3}\left(\mathbb{P}^{1}\right)$ is the twisted cubic, which is $A_{2}$-minuscule, but not in its minimal embedding.

Let $X=G / P$ with $P$ maximal, let $H$ the semi-simple part of $P$. We obtain a splitting $T_{x} X=\oplus_{p} T_{p}$, with each $T_{p}$ an irreducible $H$-module. Let $Y_{p} \subset \mathbb{P} T_{p}$ denote the closed orbit.

Proposition 2.12. The closed orbit $Y_{p}$ is contained in Base $\left|\mathbb{F F}_{X, x}^{p+1}\right|$, and there is a rational normal curve in $X$ of degree at most $p+1$, passing through $x$ with tangent vector in $Y_{p}$.

This proposition indicates that it is possible to study the $G$-homogeneous rational curves on $G / P$ of degree greater than one using the methods we use to study lines on $G / P$.

Proof. Let $P=P_{\alpha_{j}}$ and let $\beta$ be such that $m_{j}(\beta)=p$. Let $X_{\beta} \in \mathfrak{g}_{-\beta}$. Let $v \in V$ be a highest weight vector and $x=[v]$. Then $X_{\beta} v \in \hat{Y}_{p} \subset T_{p}$. By [6], lemme 7.2.5, $X_{\beta}^{p+1} v=0$ so the rational curve $\exp \left(t X_{\beta}\right) v$ is contained in $X$ and is of degree at most $p$.

These propositions stress the importance of minuscule varieties in our study. The next section is devoted to their properties.

\section{Minuscule varieties}

We explicitly describe the tangent and normal spaces to minuscule varieties $X=$ $G / P$ in $\S 3.1$ and $\S 3.3$ as $H$-modules, where $H$ is the semi-simple part of the Levi factor of $P$. In $\S 3.2$ we state an prove our main theorem that determines the fundamental forms of minuscule varieties. In $\S 3.4$ we remark on some interesting complexes obtained from the normal spaces of minuscule varieties. 


\subsection{Their tangent spaces}

We summarize characterizations and tangent space structures of minuscule varieties: Let $G$ be a simple Lie group and $P=P_{\alpha_{i}}$ a maximal parabolic subgroup. Let $G / P \subset \mathbb{P} V$ be the minimal homogeneous embedding. The following are equivalent:

1. $m_{i}(\tilde{\alpha})=1$ (the highest root $\tilde{\alpha}$ has coefficient one on the simple root $\alpha_{i}$ ),

2. $\mathfrak{p}^{u}$ is an abelian subalgebra of $\mathfrak{g}$,

3. $T=T_{[e]}(G / P)$ contains no $P$-invariant submodule,

4. $G / P$ admits an irreducible Hermitian symmetric metric with local holonomy $G$ induced from a Fubini-Study metric on $\mathbb{P} V$, and the embedding to $\mathbb{P} V$ is the smallest such embedding.

Here is a table of the $G$-minuscule varieties: there are four infinite series and two exceptional spaces.

\begin{tabular}{|c|c|c|c|c|}
\hline Name & Grassmannian & Quadric & Lagrangian Grassm. & Quadric \\
\hline Notation & $G(k, n+1)$ & $\mathbb{Q}^{2 n-1}$ & $G_{\text {Lag }}(n, 2 n)$ & $\mathbb{Q}^{2 n-2}$ \\
\hline$G$ & $A_{n}$ & $B_{n}$ & $C_{n}$ & $D_{n}$ \\
\hline$\omega$ & $\omega_{k}$ & $\omega_{1}$ & $\omega_{n}$ & $\omega_{1}$ \\
\hline $\mathcal{D}(G)$ & $0-0-0-0$ & $=$ & & \\
\hline$H$ & $A_{k-1} \times A_{n-k}$ & $B_{n-1}$ & $A_{n-1}$ & $D_{n-1}$ \\
\hline$\phi_{1}$ & $\omega_{k-1}+\omega_{k+1}$ & $\omega_{1}$ & $2 \omega_{n-1}$ & $\omega_{1}$ \\
\hline $\mathcal{D}(H)$ & $\circ \circ \bullet \bullet$ & - $\quad 0 \quad 00$ & $0-0-0-0$ & - -0 \\
\hline$T$ & $E^{*} \otimes Q$ & $E^{*} \otimes\left(E^{\perp} / E\right)$ & $S^{2} Q$ & $E^{*} \otimes\left(E^{\perp} / E\right)$ \\
\hline
\end{tabular}

\begin{tabular}{|c|c|c|c|}
\hline Name & Spinor variety & Cayley plane & $? ?$ \\
\hline Notation & $\mathbb{S}_{n}$ & $\mathbb{O P}^{2}$ & $G_{\omega}\left(\mathbb{O}^{3}, \mathbb{O}^{6}\right)$ \\
\hline$G$ & $D_{n}$ & $E_{6}$ & $E_{7}$ \\
\hline$\omega$ & $\omega_{n}$ & $\omega_{1}$ & $\omega_{7}$ \\
\hline $\mathcal{D}(G)$ & & $-0-0$ & $0-0$ \\
\hline$H$ & $A_{n-1}$ & $D_{5}$ & $E_{6}$ \\
\hline$\phi_{1}$ & $\omega_{n-2}$ & $\omega_{4}$ & $\omega_{6}$ \\
\hline $\begin{array}{c}\mathcal{D}(H) \\
\quad T\end{array}$ & $\begin{array}{lll}\Lambda^{2} E^{*} & - \\
& - & -\end{array}$ & $\begin{array}{ll}\mathcal{S}^{+} & 0 \\
& \end{array}$ & $\begin{array}{l}\mathcal{J}_{3}(\mathbb{O}) \\
\end{array}$ \\
\hline
\end{tabular}

Here $E$ and $Q$ are the tautological and quotient vector bundles on the Grassmannian or their pullbacks to the varieties in question. $\mathcal{S}^{+}$is the half spin representation of $D_{5}$, and $\mathcal{J}_{3}(\mathbb{O})$ is the space of $3 \times 3 \mathbb{O}$-Hermitian matrices, the representation $V_{\omega_{1}}$ for $E_{6}$ (see $\S 6.2$ for details). $G_{\omega}\left(\mathbb{O}^{3}, \mathbb{O}^{6}\right)$ may be interpreted as the space of $\mathbb{O}^{3}$ s in $\mathbb{O}^{6}$ that are null for an $\mathbb{O}$-Hermitian symplectic form, see [15]. 


\subsection{The strict prolongation property}

We prove our main theorem on the infinitesimal geometry of minuscule varieties.

Theorem 3.1. Let $X=G / P_{\alpha_{i}} \subset \mathbb{P} V_{\omega_{i}}$ be a minuscule variety and $x \in X$. Then for $k \geq 2$,

$$
\left|\mathbb{F F}_{x, X}^{k+1}\right|=\left|\mathbb{F F}_{x, X}^{2}\right|^{(k-1)}
$$

Remark 3.2. This result says that the leading terms of the Taylor series in local coordinates adapted to the filtration by osculating spaces, are determined by the quadratic terms in an elementary manner. In [15], we show moreover that there are no terms in the Taylor series except for the leading terms. (Minuscule varieties are the unique homogeneous varieties having this property.)

Proof. Let $v=v_{\omega_{i}} \in V_{\omega_{i}}$ be the highest weight vector, and let $T=T_{[v]} X$. We denote by $R_{k} \subset S^{k} T$ the space of relations of degree $k$, that is, the space of homogeneous polynomials $P_{k}$ of degree $k$ in the $X_{\alpha}$, with $\alpha \in \Delta_{X}$, such that $P_{k} \cdot v \in$ $\hat{T}_{[v]}^{(k-1)} X$, the $(k-1)$-st osculating space. We have the following commutative diagram, where horizontal middle long sequence and the vertical short sequences are exact:

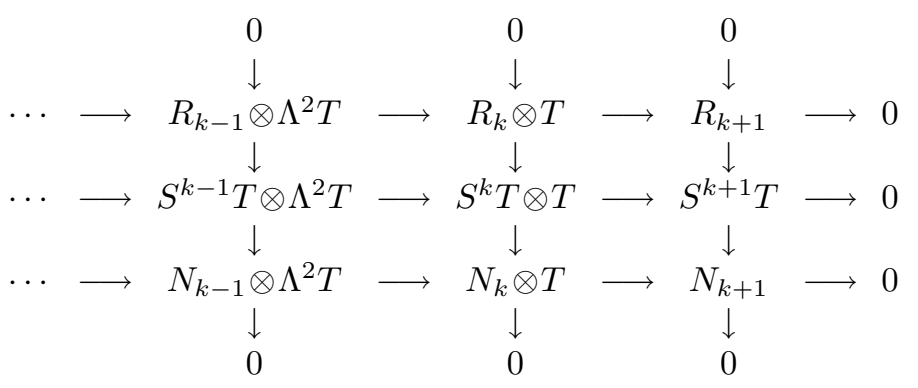

Lemma 3.3. $N_{k+1}^{*}=N_{k}^{*(1)}$ for all $k \geq 2$ if and only if the relations are generated in degree two, that is, the map $R_{k} \otimes T \rightarrow R_{k+1}$ is surjective for all $k \geq 2$.

Proof. We first note that $N_{k+1}^{*}=N_{k}^{*(1)}$ holds if and only if the sequence

$$
N_{k+1}^{*} \longrightarrow N_{k}^{*} \otimes T^{*} \longrightarrow N_{k-1}^{*} \otimes \Lambda^{2} T^{*}
$$

is exact at the middle term. This is because, by definition, $N_{k}^{*(1)}=\left(N_{k}^{*} \otimes T^{*}\right) \cap$ $S^{k+1} T^{*}$ and $S^{k+1} T^{*}$ is the kernel of the map $S^{k} T^{*} \otimes T^{*} \rightarrow S^{k-1} T^{*} \otimes \Lambda^{2} T^{*}$.

A diagram chase, using the above partially exact diagram, shows that the exactness of the dual sequence $N_{k-1} \otimes \Lambda^{2} T \longrightarrow N_{k} \otimes T \longrightarrow N_{k+1}$ is equivalent to the surjectivity of the map $R_{k} \otimes T \rightarrow R_{k+1}$. 
Now we analyze the space of relations. By [6] (Lemme 7.2.5 p. 225), the relations all come from the identities

$$
X_{\alpha_{i}}^{2} v=0 \quad \text { and } \quad X_{\beta} v=0 \text { for } m_{i}(\beta)=0 .
$$

More precisely, if $P_{k}$ is a homogeneous relation of degree $k$, there exists an identity of the following kind in $U(\mathfrak{n})$ (where $\mathfrak{n}$ is the subalgebra of $\mathfrak{g}$ generated by positive root vectors):

$$
P_{k}+Q_{<k}+\sum_{m_{i}(\beta)=0} R_{\beta} X_{\beta}+S X_{\alpha_{i}}^{2}=0,
$$

where $Q_{<k}$ is a polynomial of degree less than $k$ in the $X_{\alpha}, \alpha \in \Delta_{X}$, and the $R_{\beta}$ and $S$ are polynomials in the $X_{\gamma}, \gamma \in \Delta_{+}$. Now we fix an ordered basis of $\mathfrak{n}$, beginning first with the $X_{\beta}, \beta \neq \alpha_{i}$, such that $m_{i}(\beta)>0$, then $X_{\alpha_{i}}$, and then continuing with the $X_{\gamma}$ for which $m_{i}(\gamma)=0$. By the Poincaré-Birkhoff-Witt theorem ([6], Théorème 2.1.11 p. 69), the monomials in the $X_{\gamma}$ compatible with this order form a basis of $U(\mathfrak{n})$.

We say that a polynomial expression in the $X_{\gamma}, \gamma \in \Delta_{+}$, is well-ordered if each of its monomials is compatible with our ordered basis. We may suppose that in the identity above, all the polynomials $P_{k}, Q_{<k}, R_{\beta}$ and $S$ are well-ordered. We may even suppose that the products $R_{\beta} X_{\beta}$ are well-ordered, as if they are not, reordering them gives a sum of expressions of the same type, since the space generated by the $X_{\gamma}$ for which $m_{i}(\gamma)=0$ is stable under the Lie bracket. However, and this is the crucial point, we cannot suppose a priori that the product $S X_{\alpha_{i}}^{2}$ is also well-ordered.

The conclusion of this analysis is that all relations appear in the following way: we first chose a well-ordered monomial $X_{\beta_{1}} \cdots X_{\beta_{m}}$, with $m_{i}\left(\beta_{1}\right)=\cdots=$ $m_{i}\left(\beta_{m}\right)=0$; we reorder its product with $X_{\alpha_{i}}^{2}$, which gives an expression of the form:

$$
X_{\beta_{1}} \cdots X_{\beta_{m}} X_{\alpha_{i}}^{2}=\sum_{\gamma \delta} c_{\gamma \delta} X_{\alpha_{i}+\gamma} X_{\alpha_{i}+\delta}+C X_{2 \alpha_{i}+\beta_{1}+\cdots+\beta_{m}}+\sum_{m_{i}(\eta)=0} U_{\eta} X_{\eta},
$$

where $U_{\eta}$ is some polynomial in the $X_{\gamma}$ and $C$ is a constant. We then multiply on the left by a monomial in the $X_{\beta}$ with $m_{i}(\beta)>0$ and reorder if necessary, then we make linear combinations, and finally, we only keep the homogeneous terms of maximal degree in the resulting expression.

This doesn't seem very enlightening, but since $X$ is a minuscule variety, if $m_{i}(\beta)=m_{i}(\gamma)=1$, then $X_{\beta}$ and $X_{\gamma}$ commute. So the above relation simplifies to an expression of the form

$$
X_{\beta_{1}} \cdots X_{\beta_{m}} X_{\alpha_{i}}^{2}=\sum_{\gamma \delta} c_{\gamma \delta} X_{\alpha_{i}+\gamma} X_{\alpha_{i}+\delta}+\sum_{m_{i}(\eta)=0} U_{\eta} X_{\eta} .
$$

Moreover, the relations are then obtained by multiplying the sums $\sum_{\gamma \delta} c_{\gamma \delta} X_{\alpha_{i}+\gamma} X_{\alpha_{i}+\delta}$ by monomials in the $X_{\alpha_{i}+\eta}$, which need no reordering; and 
finally, the resulting expression is necessarily homogeneous, since we can assume that all its monomials have the same total weight.

This means in particular that all relations are deduced from the degree two relations

$$
\sum_{\gamma \delta} c_{\gamma \delta} X_{\alpha_{i}+\gamma} X_{\alpha_{i}+\delta}=0
$$

by simple polynomial multiplication in $T$. Thus the maps $R_{2} \otimes S^{k-1} T \rightarrow R_{k+1}$ are surjective for $k \geq 2$, which implies surjectivity of $R_{k} \otimes T \rightarrow R_{k+1}$.

\subsection{Their normal spaces}

An interesting property of $G$-minuscule varieties is that the irreducibility of the tangent space propagates to the irreducibility of all normal spaces. Indeed, the normal spaces and fundamental forms of the minuscule varieties are as follows:

Proposition 3.4. The tangent space T, and the normal spaces $N_{j}$, with $2 \leq j \leq l$, of the classical irreducible minuscule varieties $X$ are given by the following table:

\begin{tabular}{||c|c|c|c|c||}
\hline$X$ & $G(k, n)$ & $G_{\text {Lag }}(n, 2 n)$ & $\mathbb{S}_{2 n}$ & $\mathbb{Q}^{n}$ \\
$G$ & $S L_{n}$ & $S p_{2 n}$ & $\operatorname{Spin}_{2 n}$ & $S O_{n+2}$ \\
$H$ & $S L_{k} \times S L_{n-k}$ & $S L_{n}$ & $S L_{n}$ & $S O_{n}$ \\
$T$ & $W_{\omega_{k-1}+\omega_{k+1}}=E^{*} \otimes Q$ & $W_{2 \omega_{1}}=S^{2} U$ & $W_{\omega_{n-2}}=\Lambda^{2} U$ & $W_{\omega_{2}}$ \\
$N_{j}$ & $W_{\omega_{k-j}+\omega_{k+j}}=\Lambda^{j} E^{*} \otimes \Lambda^{j} Q$ & $W_{2 \omega_{j}}=S_{2 \ldots 2} U$ & $W_{\omega_{n-2 j}}=\Lambda^{2 j} U$ & $\mathbb{C}$ \\
$l$ & $\min (k, n-k)$ & $n$ & {$\left[\frac{n}{2}\right]$} & 2 \\
\hline
\end{tabular}

For the two exceptional irreducible minuscule varieties, we have the following table:

\begin{tabular}{||c|c|c|c|c|c||}
\hline$X$ & $G$ & $H$ & $T$ & $N_{2}$ & $N_{3}$ \\
\hline $\mathbb{O P}^{2}$ & $E_{6}$ & $\operatorname{Spin}_{10}$ & $W_{\omega_{2}}$ & $W_{\omega_{6}}$ & 0 \\
\hline$G_{\omega}\left(\mathbb{O}^{3}, \mathbb{O}^{6}\right)$ & $E_{7}$ & $E_{6}$ & $W_{\omega_{6}}$ & $W_{\omega_{1}}$ & $\mathbb{C}$ \\
\hline
\end{tabular}

The fundamental forms may be described explicitly as follows:

For a non-degenerate quadric $\mathbb{Q}^{n}$, the second fundamental form is a nondegenerate quadratic form with base locus a smooth quadric $\mathbb{Q}^{n-2}$.

For the respective cases $G(k, v), G_{\omega}(k, V), \mathbb{S}, G_{\omega}\left(\mathbb{O}^{3}, \mathbb{O}^{6}\right), T$ is a (subset) of a matrix space, respectively $T=E^{*} \otimes Q, S^{2} E^{*}, \Lambda^{2} E^{*}, \mathcal{J}_{3}(\mathbb{O})$. In all but $\mathbb{S}$, the last fundamental form is the set of maximal minors (the determinant for $S^{2} E^{*}$ and $\mathcal{J}_{3}(\mathbb{O})$ ), and the lower fundamental forms are just the successive Jacobian ideals. For $\mathbb{S}$, the last form is the Pfaffian (since the determinant is a square) and 
the other forms are the successive Jacobian ideals, which are the Pfaffians of the minors centered about the diagonal.

For the $\mathbb{O P}^{2}$ case, let $V=\mathbb{C}^{10}$. Then $T=S_{+}(V)$ is a half-spin representation, and $N_{2}$ is the vector representation $V$. The half-spin representations $S_{+}$and $S_{-}$can be constructed as the even and odd parts of the exterior algebra of a null 5-plane $E$ in $V$ (see e.g. [10]): $S_{+}, S_{-}$are dual to one another, the wedge product giving a perfect pairing $S_{+} \otimes S_{-} \longrightarrow \Lambda^{5} E=\mathbb{C}$. Moreover, the full exterior algebra of $E$ is a module over the Clifford algebra of $V$. If $F$ is a complementary null 5-plane of $E$, then $E$ acts on $S_{+}$by exterior multiplication, $F$ by interior multiplication, and this action of $V=E \oplus F$ extends to the whole Clifford algebra. In particular, there is a natural map from $V$ to $\operatorname{End}\left(S_{-}, S_{+}\right) \simeq S_{+} \otimes S_{+}$. The transpose of the symmetric part of this morphism is the second fundamental form.

Alternatively, identifying $S_{+}(V)=\mathbb{O} \oplus \mathbb{O}$ (see [10]) with octonionic coordinates $u, v$, we have $\left|\mathbb{F F}_{X, x}^{2}\right|=\{u \bar{u}, u \bar{v}, v \bar{v}\}$ where, considering $\mathbb{O}$ as an eight dimensional vector space over $\mathbb{C}$, the middle equation is eight quadrics.

Remark 3.5. Note that in all cases, the only $H$-orbit closures in $\mathbb{P} T_{1}$ are the secant varieties. This actually characterizes the minuscule varieties, see [15]. A special case of this phenomenon is observed in [22]. Note that this property also allows one to easily classify the $G$-orbits in $\tau(X)$ when $X$ is minuscule. See [14] for examples.

Corollary 3.6. Let $X$ be a minuscule variety, and let $x \in X$. Then

$$
\text { Base }\left|\mathbb{F}_{X, x}^{k}\right|=\sigma_{k-1}\left(\text { Base }\left|\mathbb{F}_{X, x}^{2}\right|\right) \text {. }
$$

Moreover, $\left|\mathbb{F F}_{X, x}^{k}\right|=I_{k}\left(\right.$ Base $\left.\left|\mathbb{F F}_{X, x}^{k}\right|\right)$.

Proof of the corollary. Immediate from our explicit descriptions of the fundamental forms.

Proof of the proposition. For each of these varieties, and each integer $j$, we check that there is a unique irreducible $H$-module which is a component of both $S^{j} T$ and of the restriction $\operatorname{Res}_{H}^{G} V_{\omega_{i}}$. Then $N_{j}$ must be this $H$-module. For an ordinary Grassmannian $G(k, n)=G(k, V), T=E^{*} \otimes Q$, where $E$ is the tautological subbundle and $Q=V / E$ the quotient bundle. Its symmetric powers are given by the Cauchy formula ([21], p. 33)

$$
S^{j} T=\bigoplus_{|\lambda|=j} S_{\lambda} E^{*} \otimes S_{\lambda} Q
$$

the sum is over all partitions $\lambda$ with the sum of its parts $|\lambda|$ equal to $j$. We have

$$
\operatorname{Res}_{H}^{G} \Lambda^{k} V=\Lambda^{k}(E \oplus Q)=\bigoplus_{h \geq 0} \Lambda^{h} E^{*} \otimes \Lambda^{h} Q=\bigoplus_{h \geq 0} W_{\omega_{k-h}+\omega_{k+h}}
$$


since $\operatorname{rank}(E)=k$. The only common component of these two decompositions is $\Lambda^{j} E^{*} \otimes \Lambda^{j} Q=W_{\omega_{k-j}+\omega_{k+j}}$. The case of Lagrangian Grassmannians is similar. Here $Q \simeq E^{*}, T=S^{2} E^{*}$ and we use the formula ([21], p. 45)

$$
S^{j} T=\bigoplus_{|\lambda|=j} S_{2 \lambda} E^{*}
$$

We compute the decomposition

$$
\operatorname{Res}_{H}^{G} V_{\omega_{n}}=\bigoplus_{h \geq 0} S_{\underbrace{2 \ldots 2}_{h}} E^{*}=\bigoplus_{h \geq 0} W_{2 \omega_{h}},
$$

and the conclusion follows as above. On spinor varieties, $Q \simeq E^{*}$ again, $T=\Lambda^{2} E^{*}$ and we use the formula ([21], p. 46)

$$
S^{j} T=\bigoplus_{|\lambda|=j} S_{\lambda(2)} E^{*}
$$

where if $\lambda=\left(\lambda_{1}, \ldots, \lambda_{m}\right)$, then $\lambda(2)=\left(\lambda_{1}, \lambda_{1}, \ldots, \lambda_{m}, \lambda_{m}\right)$. Finally, the case of quadrics is immediate since they are hypersurfaces.

For exceptional minuscule varieties the same argument goes through, except that we use the LiE package [20], or Littelmann paths, instead of the above classical decomposition formulas.

\subsection{Algebraic structures induced by infinitesimal geometry}

We remark on some consequences of the strict prolongation property for minuscule varieties.

Proposition 3.7. Let $X^{n} \subset \mathbb{P}^{n+a}$ be a variety such that strict prolongation holds at $x \in X$. Let $N_{j}=N_{j, x} X$. Then there are natural maps

$$
N_{i}^{*} \otimes N_{j}^{*} \rightarrow N_{i+j}^{*}
$$

Proof. The maps are the restrictions of the symmetrization maps $S^{i} T^{*} \otimes S^{j} T^{*} \rightarrow$ $S^{i+j} T^{*}$ and the image is assured to lie in $N_{i+j}^{*}$ by the strict prolongation property.

Corollary 3.8. Let $X=G / P \subset \mathbb{P V}$ be a minuscule variety. Let $H$ be the semisimple part of $P$. Then there is a natural structure of a graded $H$-algebra on $V$.

In the case of Grassmannians, the algebra structure on $\Lambda^{k} V$ is given by the multiplication of minors. For Lagrangian Grassmannians, it is related to the multiplication of Pfaffians. The exceptional cases are related to certain exceptional algebraic structures introduced by Freudenthal, that we meet again in $\S 6$. For example, consider the minuscule variety of $E_{7}$ : this is a 27-dimensional subvariety 
of the projectivization of the minimal representation $V$ of $E_{7}$, whose dimension is 56. As an $H$-module, we have

$$
V=V_{0} \oplus V_{1} \oplus V_{2} \oplus V_{3}=\mathbb{C} \oplus \mathcal{J}_{3}(\mathbb{O}) \oplus \mathcal{J}_{3}(\mathbb{O})^{*} \oplus \mathbb{C},
$$

where $\mathcal{J}_{3}(\mathbb{O})$ denotes the exceptional Jordan algebra of $3 \times 3 \mathbb{O}$-Hermitian matrices. The group $H=E_{6}$ is realized as the subgroup of $G L\left(\mathcal{J}_{3}(\mathbb{O})\right)$ preserving the cubic form defined by the determinant. Its polarization defines the map $V_{1} \otimes V_{1} \rightarrow V_{2}$. The map $V_{1} \otimes V_{2} \rightarrow V_{3}$ is just the evaluation.

Another consequence of the strict prolongation property at a point of any variety is the appearance of Koszul complexes:

Corollary 3.9. Let $X^{n} \subset \mathbb{P}^{n+a}$ be a variety such that strict prolongation holds at $x \in X$. Let $N_{j}=N_{j, x} X$. Then there is a Koszul complex:

$$
\cdots \longrightarrow N_{j-1}^{*} \otimes \Lambda^{k+1} T^{*} \longrightarrow N_{j}^{*} \otimes \Lambda^{k} T^{*} \longrightarrow N_{j+1}^{*} \otimes \Lambda^{k-1} T^{*} \longrightarrow \cdots
$$

induced by the maps $T^{*} \otimes N_{j}^{*} \rightarrow N_{j+1}^{*}\left(\right.$ recall that $\left.T^{*}=N_{1}^{*}\right)$.

If $N_{j}^{*}$ is replaced by the space of sections $\Gamma\left(X, \mathcal{O}_{X}(j)\right)$ for a subvariety $X \subset \mathbb{P} T$, the homology of the corresponding Koszul complexes compute the syzygies of $X$ [8].

For a classical minuscule variety $X$, there is a strange relation between the complexes constructed from their normal spaces, and the Koszul complexes computing the syzygies of another minuscule variety $Z$. Indeed, we obtain this second family of complexes from the first, by a natural involution on the set of highest weights of irreducible $L$-modules.

For $L=G L_{n}$, this involution is defined in the following way: to the Schur power $S_{\lambda}$ we associate $S_{\lambda^{*}}$, where $\lambda^{*}$ is the conjugate partition of $\lambda$, obtained by symmetry along the main diagonal of its diagram (which actually defines a bijection between partitions inscribed in a $k \times(n-k)$ rectangle, and partitions inscribed in a $(n-k) \times k$ rectangle). The complex associated to our example above is therefore

$$
\cdots \longrightarrow S^{j} E \otimes S^{j} F \otimes \Lambda^{k}(E \otimes F) \longrightarrow S^{j+1} E \otimes S^{j+1} F \otimes \Lambda^{k-1}(E \otimes F) \longrightarrow \cdots
$$

In small degrees, for $X=G(k, n), G_{\text {Lag }}(n, 2 n), \mathbb{S}_{n}$, we obtain the Koszul complexes associated to $Z=\mathbb{P}^{n-k-1} \times \mathbb{P}^{k-1}, G(2, n), v_{2}\left(\mathbb{P}^{n-1}\right)$ respectively. (Note that $Y_{1}=\mathbb{P}^{k-1} \times \mathbb{P}^{n-k-1}, v_{2}\left(\mathbb{P}^{n-1}\right), G(2, n)$ respectively.)

\section{Linear spaces on homogeneous varieties}

In this section we explicitly describe the lines through a point of a homogeneous variety $X=G / P \subset \mathbb{P} V$, the Fano variety parametrizing all lines on $X$ (in $\S 4.2$ ), as well as the Fano varieties parametrizing higher dimensional linear spaces on $X$ 
in $§ 4.3$. These parameter spaces all come equipped with natural embeddings to projective spaces whose associated vector spaces are $G$-modules as described in §4.4. We give an amusing recipe for recovering the Dynkin diagram of $G$ from second fundamental form data in $\S 4.5$. We begin, in $\S 4.1$ with a construction due to Tits that is essential to our work.

\subsection{Tits fibrations}

Let $G$ be a simple Lie group, let $S, S^{\prime}$ be two subsets of the sets of simple roots of $G$. Consider the diagram

$$
X=G / P_{S}{ }^{\pi \swarrow^{G / P_{S \cup S^{\prime}}} \searrow \pi^{\prime}} X^{\prime}=G / P_{S^{\prime}}
$$

Let $x^{\prime} \in X^{\prime}$ and consider $Y:=\pi\left(\pi^{\prime-1}\left(x^{\prime}\right)\right) \subset X$. Then $X$ is covered by such varieties $Y$. Tits shows in [24] that $Y=H / Q$ where $\mathcal{D}(H)=\mathcal{D}(G) /\left(S \backslash S^{\prime}\right)$, and $Q \subset H$ is the parabolic subgroup corresponding to $S^{\prime} \backslash S$. He calls such subvarieties $Y$ of $X, L$-subvarieties, and $Y$ the shadow of $x^{\prime}$.

Example 4.1. For $X=D_{n} / P_{3}$ and $X^{\prime}=D_{n} / P_{n}$, we read off the diagram below that $Y=G(3, n)$.

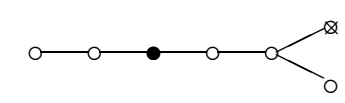

\subsection{Lines}

Let $\mathcal{D}=\mathcal{D}(G)$ be the Dynkin diagram of a complex simple Lie group $G$. We identify the nodes of $\mathcal{D}$ with the set of simple roots with respect to a choice of maximal torus $T$ and Borel subgroup $B$ which we fix once and for all. Let $\alpha \in \mathcal{D}$. Let $N(\alpha)=\{\beta \in \mathcal{D} \mid(\alpha, \beta)<0\}$ denote the neighbors of $\alpha$, the simple roots connected to $\alpha$ by an edge in $\mathcal{D}$.

Proposition 2.5 implies the following (with the same notations):

Corollary 4.2. Let $X=G / P$, with $G$ simple and $P$ a maximal parabolic subgroup. Let $Y_{1} \subset \mathbb{P} T_{1}$ be the closed orbit. Then $Y_{1}$ is isomorphic to the shadow of a point $x \in X$ on the space $X^{\prime}=G / P^{\prime}$ of G-lines in $X$.

Here $P^{\prime}$ is the parabolic subgroup of $G$ defined by the neighbors of the root defining $P$.

We will see that if we consider the minimal homogeneous embedding $X \subset \mathbb{P} V$, these $G$-Tits lines are linearly embedded. We first need to recall a few basic facts on the Picard group of a rational homogeneous space. 
It a classical fact, due to Chevalley, that $\operatorname{Pic}(G / B)=H^{2}(G / B, \mathbb{Z})=P$, the weight lattice ([3], Exposé 15). More generally, $\operatorname{Pic}\left(G / P_{S}\right)=H^{2}\left(G / P_{S}, \mathbb{Z}\right)=$ $P(S)$, the sublattice generated by the fundamental weights $\omega_{i}$ dual to the roots $\alpha_{i} \in S$ (or rather to the corresponding coroots). Dually, $H_{2}\left(G / P_{S}, \mathbb{Z}\right) \simeq \check{R}(S)$, the lattice generated by the coroots $\check{\alpha}_{i}$ to the roots $\alpha_{i} \in S$, with the obvious pairing with $P(S)$. Each class $\check{\alpha}_{j}$ can be realized geometrically by considering the double fibration

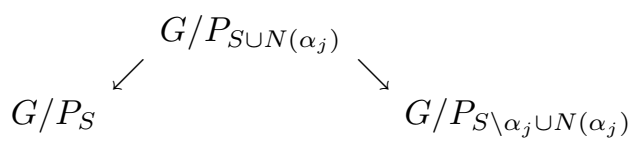

Indeed, the shadow on $G / P_{S}$ of a point in $G / P_{S \backslash \alpha_{j} \cup N\left(\alpha_{j}\right)}$ is a rational curve, on which a line bundle $L_{\lambda}$ defined by a weight $\lambda \in P(S)$ has degree $\left\langle\lambda, \check{\alpha}_{j}\right\rangle$ (see [5], Lemme 2 p. 58).

In particular, suppose that $G / P_{S}$ is embedded in some $\mathbb{P} V_{\lambda}$ by a very ample line bundle $L_{\lambda}$, where $\lambda=\sum_{i \in S} l_{i} \omega_{i}$, and contains a line of $\mathbb{P} V_{\lambda}$ whose homology class is $\check{\beta}=\sum_{i \in S} m_{i} \check{\alpha}_{i}$. Then $\sum_{i \in S} l_{i} m_{i}=1$, which implies that $\check{\beta}=\check{\alpha}_{j}$ for some $j \in S$ with $l_{j}=1$. Moreover, the variety $F_{1}^{j}(X)$ of these $\alpha_{j}$-lines on $X$ is independent of the $\lambda$ with $l_{j}=1$ chosen. Note that it contains $G / P_{S \backslash \alpha_{j} \cup N\left(\alpha_{j}\right)}$.

Theorem 4.3. Let $S \subseteq \mathcal{D}$, consider $X=G / P_{S}$ in its minimal homogeneous embedding. Then

1. $F_{1}(X)=\coprod_{j \in S} F_{1}^{j}(X)$, where $F_{1}^{j}(X)$ is the space of lines of class $\check{\alpha}_{j} \in$ $H_{2}\left(G / P_{S}, \mathbb{Z}\right)$.

2. If $\alpha_{j}$ is not an exposed short root, then $F_{1}^{j}(X)=G / P_{S \backslash \alpha_{j} \cup N\left(\alpha_{j}\right)}$.

3. If $\alpha_{j}$ is an exposed short root, then $F_{1}^{j}(X)$ is the union of two $G$-orbits, an open orbit and its boundary $G / P_{S \backslash \alpha_{j} \cup N\left(\alpha_{j}\right)}$.

Assertions 1. and 2. are rephrasings of results in [4], which were published just after the first version of this paper was written (but note that Cohen and Cooperstein work over an arbitrary field). Assertion 3. and its proof below were communicated to us by an anonymous referee (our original proof contained some case by case arguments).

In $\S 6$ we give explicit descriptions of the open orbits of 3. for each short root.

Proof. The argument, proceeds in three steps: first we give a criterion for identifying distinct orbits in $F_{1}^{j}(X)$; up to the action of $W_{S}$, the subgroup of the Weyl group $W$ generated by the simple reflections $s_{i}, i \notin S$, there is a unique $T$-fixed point in each $G$-orbit passing through a base point $x \in X$, where $T$ denotes the maximal torus in $G$. We then show that there is a unique tangent direction in $T_{j 1}$ corresponding to a line up to $W_{S}$-equivalence, and finally, if $\alpha_{j}$ is an exposed short root with $-\left(\alpha, \alpha_{j}\right)=2$ (resp. 3$)$, then there is a unique vector in $T_{j 2}$ (resp. $T_{j 3}$ ) modulo $W_{S}$-equivalence corresponding to a $T$-fixed line. Finally we show that the orbit of the $T_{j 2}\left(\operatorname{resp} . T_{j 3}\right)$ line is not closed. 
We first observe that every $G$-orbit in $F_{1}^{j}(X)$ contains a $T$-fixed line. Indeed, if $l$ is an $\alpha_{j}$-line, by homogeneity we can suppose it contains the base point $x=\left[P_{S}\right] \in$ $X$. If $y \in l$ is another point, $y$ lies in a unique Bruhat cell $B(w)=\left\{w x h^{-1} \mid h \in\right.$ $B\}, w \in W$. Then there exists $h \in B$ such that $h(y)=w(x)$, and since $h(x)=x$, the line $l^{\prime}=\overline{x w(x)}$ is $T$-stable in $G l$.

By $T$-stability, the tangent direction to $l^{\prime}$ at $x$ must be a $\mathfrak{g}_{-\alpha}$ for some $\alpha \in \Delta_{X}$. The orbit through $x$ of the $S L_{2}$ corresponding to $\alpha$ is $l$, and thus $\langle\lambda, \check{\alpha}\rangle=1$ because $\lambda$ is also the highest weight of the $S L_{2}$-module $\hat{l} \simeq \mathbb{C}^{2}$.

Write $\alpha=p \alpha_{j}+\gamma$ with $m_{j}(\gamma)=0$. We have

$$
1=\langle\lambda, \check{\alpha}\rangle=p \frac{\left(\alpha_{j}, \alpha_{j}\right)}{(\alpha, \alpha)}+\Sigma_{i \neq j} l_{i}\left\langle\omega_{i}, \check{\alpha}\right\rangle .
$$

Since the equality holds for any choices of coefficients $l_{i}$, we must have $\left\langle\omega_{i}, \check{\alpha}\right\rangle=0$ for all $i \in S \backslash j$. Thus $(\alpha, \alpha)=p\left(\alpha_{j}, \alpha_{j}\right)$ and we have two cases.

If $\alpha_{j}$ is not an exposed short root, then $p=1$ and by Lemma 4.4 below, $\alpha$ is $W_{S}$-conjugate to $\alpha_{j}$. There is therefore a unique $G$-orbit in $F_{1}^{j}(X)$.

If $\alpha_{j}$ is an exposed short root, then either $p=1$ and $\alpha$ is short, or $p>1$ and $\alpha$ is long. Then Lemma 4.4 and Lemma 4.6 below imply that there are at most two $G$-orbits in $F_{1}^{j}(X)$.

The following lemma was communicated to us anonymously.

Lemma 4.4. Let $\beta \in \Delta_{X}$ have the same length as $\alpha_{i}$, and $m_{i}(\beta)=1$. Then there exists $w \in W_{S}$ with $w \alpha_{i}=\beta$.

Proof. We use induction on the height of $\beta$, i.e., the sum of its coefficients in its decomposition on simple roots. Suppose that $\beta \neq \alpha_{i}$, and $\left(\beta, \alpha_{j}\right) \leq 0$ for all $j \neq i$. Then $\left(\beta, \alpha_{i}\right) \geq(\beta, \beta)>0$, thus $n\left(\beta, \alpha_{i}\right)=1$ and $s_{i}(\beta)=\gamma$, where $s_{i}(\beta)=\beta-\left\langle\beta, \check{\alpha}_{i}\right\rangle \alpha_{i}$. Therefore, since $\beta, \gamma$ and $\alpha_{i}$ have the same length, we get $-1=n\left(\gamma, \alpha_{i}\right)=n\left(\alpha_{i}, \gamma\right)$, hence $n(\beta, \gamma)=1$ and $(\beta, \gamma)>0$.

We can therefore let $k \neq i$ such that $\left(\beta, \alpha_{k}\right)>0$. Then the root $s_{k}(\beta)$ verifies the same assumptions as $\beta$, but its height is smaller, and we conclude the proof by induction.

Lemma 4.5. $\alpha_{j}$ is an exposed short root iff there exists $\alpha \in \Delta_{+} \backslash S$ such that $\left|n\left(\alpha, \alpha_{j}\right)\right|>1$.

Proof. If $\alpha_{j}$ is not an exposed short root, $n\left(\alpha, \alpha_{j}\right) \neq 0$ implies that $\alpha$ and $\alpha_{j}$ have the same length, hence $\left|n\left(\alpha, \alpha_{j}\right)\right|=\left|n\left(\alpha_{j}, \alpha\right)\right| \leq 1$. Now say $\alpha_{j}$ is an exposed short root. Then there exists a long root $\alpha$ supported outside $S$ such that $\left(\alpha, \alpha_{j}\right) \neq 0$. Thus $\left|n\left(\alpha, \alpha_{j}\right)\right|>\left|n\left(\alpha_{j}, \alpha\right)\right|=1$.

Lemma 4.6. If $\alpha_{j}$ is an exposed short root, then any pair of long roots of the form $p \alpha_{j}+\gamma$, where $\gamma$ is supported outside $S$, are conjugate in $W_{S}$. 
Proof. If $p=3$, we are in the $G_{2}$ case which is clear. So assume $p=2$. Let $\delta=2 \alpha_{j}+\gamma, \gamma$ supported outside $S$.

Assume $\delta$ is such that $\left(\delta, \alpha_{i}\right) \leq 0$ for each $i \neq j$ with $m_{i}(\delta) \neq 0$. Then $\left(\delta, \alpha_{j}\right) \geq(\delta, \delta)>0$. Since $\alpha_{j}$ is short, we have $n\left(\delta, \alpha_{j}\right)=2$, so $s_{j}(\delta)=\gamma$ and $\gamma$ is a long root. Also, $n\left(\alpha_{j}, \delta\right)=1$, so that $n\left(\delta, \alpha_{i}\right)=0$ for all $i \neq j$ with $m_{j}(\delta) \neq 0$. Thus $\delta$ is the highest root with support in the subdiagram $\operatorname{supp}(\delta)$. But $n\left(\delta, \alpha_{j}\right)=2$, therefore this subdiagram must be of type $C_{r+2}$ for some $r \geq 0$. Since $j$ is an end of it, it is uniquely determined, and $\delta$ is a uniquely determined $\operatorname{root} \delta_{0}$.

Thus if $\delta \neq \delta_{0}$, there exists $i \neq j, \alpha_{i} \in \mathcal{D} \backslash S$, with $\left(\delta, \alpha_{i}\right)>0$. And we can then proceed by induction on the root $s_{i}(\delta)$, whose height is smaller than that of $\delta$.

Finally we show that when $\alpha_{j}$ is an exposed short root, the orbit corresponding to a long root $\alpha$ is not closed. The case of $G_{2}$ is easy (see $\S 6.1$ ), so assume $p=2$ and, using the notation of lemma 4.5, take $\alpha=\delta_{0}$. The corresponding line is $l_{\alpha}=\overline{v_{\lambda} v_{\lambda-\alpha}}$, where $v_{\lambda}$ is a highest weight vector in $V_{\lambda}$ and $v_{\lambda-\alpha}=X_{-\alpha} v_{\lambda}$, $X_{-\alpha} \in \mathfrak{g}_{-\alpha}$. Let us compute the tangent space $T_{\alpha}$ at $l_{\alpha}$ of its $G$-orbit. For $X \in \mathfrak{g}$, we have

$$
X\left(v_{\lambda} \wedge v_{\lambda-\alpha}\right)=X v_{\lambda} \wedge v_{\lambda-\alpha}+v_{\lambda} \wedge X X_{-\alpha} v_{\lambda} .
$$

If $X \in \mathfrak{g}_{-\beta}, \alpha \neq \beta \in \Delta_{X}$, then $X v_{\lambda} \wedge v_{\lambda-\alpha}$, and therefore also $X\left(v_{\lambda} \wedge v_{\lambda-\alpha}\right)$, are non zero. If $X \in \mathfrak{p}_{S}$, then $X v_{\lambda}=0$, hence $X\left(v_{\lambda} \wedge v_{\lambda-\alpha}\right)=v_{\lambda} \wedge\left[X, X_{-\alpha}\right] v_{\lambda}$. If $X$ is a root vector, this is non zero if and only if $X \in \mathfrak{g}_{\alpha-\beta}$, with $\beta \in \Delta_{X}$. This implies that, as a $T$-module,

$$
T_{\alpha}=\bigoplus_{\beta \in \Gamma} \mathfrak{g}_{-\beta}, \quad \Gamma=\left(\Delta_{X}-\{\alpha\}\right) \cup\left\{\beta-\alpha \in \Delta, \beta \in \Delta_{X}\right\} .
$$

(Note that since $\alpha$ is a long root, we also have $\Gamma=\left(\Delta_{X}-\{\alpha\}\right) \cup s_{\alpha}\left(\Delta_{X}-\{\alpha\}\right)$.)

To prove that the $G$-orbit of $l_{\alpha}$ is not closed, we need to prove that its stabilizer cannot contain a Borel subgroup, and for this it is enough to exhibit a root $\beta \in \Gamma$ such that $-\beta$ also belongs to $\Gamma$. But this is easy: indeed, recall that the subdiagram of $\mathcal{D}$ supporting $\alpha$ is of type $C_{r+2}$, and that $\alpha$ is the corresponding highest root, that is $\alpha=2 \alpha_{j}+\cdots+2 \alpha_{r+j}+\alpha_{r+j+1}$ for a suitable numbering of the simple roots. Then we can take $\beta=\alpha_{j}+\cdots+\alpha_{r+j}+\alpha_{r+j+1} \in \Delta_{X}$, since $-\beta+\alpha=$ $\alpha_{j}+\cdots+\alpha_{r+j} \in \Delta_{+}$.

Remark 4.7. This proof gives a formula for the dimension of the open orbit of lines in the case of an exposed short root, namely the cardinality of $\Gamma=\left(\Delta_{X}-\right.$ $\{\alpha\}) \cup s_{\alpha}\left(\Delta_{X}-\{\alpha\}\right)$.

On the infinitesimal level, $C_{x} \subset \mathbb{P} T_{x} X$, the set of tangent directions to lines on $X$ passing through $x$, is a union of disjoint varieties, one component $C_{x}^{\alpha}$ for each possible class $\alpha$ of lines. The proof of the preceding theorem implies the following: 
Theorem 4.8. Let $G$ be a complex simple Lie group, let $S$ be a subset of the simple roots. Let $\alpha \in S$. Let $\mathcal{D}(H)$ be the components of $(\overline{\mathcal{D}(G) \backslash S}) \backslash \alpha$ containing an element of $N(\alpha)$, where by $\overline{\mathcal{D}(G) \backslash S}$ we mean $\mathcal{D}(G) \backslash S$ plus any nodes of $S$ attached to a node of $\mathcal{D}(G) \backslash S$. Let $C_{x}^{\alpha} \subset \mathbb{P} T_{x} X$ denote the class of $\alpha$-lines through $x$.

1. If $\alpha$ is not an exposed short root, then $C_{x}^{\alpha} \simeq H / P_{N(\alpha)}$.

2. If $\alpha$ is an exposed short root, then $C_{x}^{\alpha}$ is a union of an open $P_{S}$-orbit and its boundary $H / P_{N(\alpha)}$.

With the notations of $\S 2$, the closed $P_{S}$-orbit in $C_{x}^{\alpha_{j}}$ is $Y_{1}^{j}$, and the open orbit is $P_{S} Y_{2}^{j}$. The cases of $C_{x}^{\alpha}$ for exposed short roots are described explicitly on a case by case basis in $\S 6$.

\subsection{Linear spaces of higher dimension}

A $k$-plane in $X$ must come from a linear $\mathbb{P}^{k-1}$ in some $C_{x}^{\alpha}$. We call such a $\mathbb{P}^{k}$, of class $\alpha$, and let $F_{k}^{\alpha}(X)$ denote the variety parametrizing the $\alpha$-class $\mathbb{P}^{k}$ 's on $X . F_{k}^{\alpha}(X)$ may have several components. The space of $\mathbb{P}^{k}$ 's in $X, F_{k}(X)$, is the disjoint union of the $F_{k}^{\alpha}(X)$ 's.

If $\alpha=\alpha_{j} \in S$ is not an exposed short root, it follows from Theorem 4.3 that the projection $G / P_{S} \rightarrow G / P_{S \backslash j}$ is constant on each $\alpha$-line, hence it is also constant on each $\alpha$-class $\mathbb{P}^{k}$. It follows that the space of $\alpha$-class $\mathbb{P}^{k}$ 's is a fibration over $G / P_{S \backslash j}$, and to determine the fiber, we can restrict to the subdiagram of $\mathcal{D}$ consisting in the connected component of $\alpha_{j}$ in $\mathcal{D} \backslash(S \backslash j)$. In particular, we are reduced to the case of a maximal parabolic subgroup, corresponding to a non-short root. Then we know that $C_{x}=Y_{1}$ is a minuscule variety, so it is again a homogeneous space of type $H / Q$ with $Q$ a maximal parabolic subgroup corresponding to a long root, or possibly a product of such spaces. We can therefore apply Theorem 4.3 to $Y_{1}$ to describe its lines, which gives $\mathbb{P}^{2}$ 's in the original space, and so on. The conclusion is that, not only $\mathbb{P}^{1}$ 's, but all linear spaces can be described in terms of Tits' geometries.

Theorem 4.9. Let $G$ be a simple group and let $X=G / P_{S} \subset \mathbb{P} V$ be a rational homogeneous variety in its minimal homogeneous embedding.

If $\alpha \in S$ is not an exposed short root, then for all $k, F_{k}^{\alpha}(X)$ is the disjoint union of homogeneous varieties $G / P_{\Sigma \beta_{j}}$ where $\left\{\beta_{j}\right\} \subset \Delta_{+}$is a minimal set of positive roots such that the component of $\mathcal{D}(G) \backslash\left\{\beta_{j}\right\}$ containing $\alpha$ is isomorphic to $\mathcal{D}\left(A_{k}\right)$, intersects $S$ only in $\alpha$, and $\alpha$ is an extremal node of this component.

Corollary 4.10. Let $G$ be a simple Lie group, let $S \subset \mathcal{D}(G)$, let $\alpha \in S$ with $\alpha$ not exposed short. Let $X=G / P_{S}$ be the corresponding homogeneous variety in a homogeneous embedding such that there are $\check{\alpha}$-lines. Suppose that the longest of 
the chains of type $A$ in $\mathcal{D}(G)$ beginning at $\alpha$ and containing no other element of $S$, is isomorphic to $\mathcal{D}\left(A_{n}\right)$. Then the largest linear space of class $\alpha$ on $X$ is a $\mathbb{P}^{n}$.

Example 4.11. Consider the case of $G / B \subset \mathbb{P} V_{\lambda}, \lambda=\omega_{1}+\cdots+\omega_{r}$, the sum of the fundamental weights. There are no unexposed short roots, so $C_{x}$ is the union of $r$ points, $F_{1}(X)=\coprod_{j} G / P_{\mathcal{D} \backslash \alpha_{j}}$ and $F_{k}(X)=\emptyset$ for $k \geq 2$.

Example 4.12. In the case of $D_{n} / P_{n}$, we have a unique family of lines, parametrized by isotropic subspaces of dimension $n-2, D_{n} / P_{n-2}$ and a unique family of two planes parametrized by the $Q$-isotropic flag variety $D_{n} / P_{n-3, n-1}$. There are two families of $\mathbb{P}^{3}$ 's, namely $D_{n} / P_{n-3}$ and $D_{n} / P_{n-4, n-1}$. For $4 \leq k \leq n-1$ there is a unique family of $\mathbb{P}^{k}$ 's, namely $D_{n} / P_{n-k-1, n-1}$.

Example 4.13. The largest linear space on $E_{n} / P_{1}$ is a $\mathbb{P}^{n-1}$, via the chain terminating with $\alpha_{n}$, so $E_{n} / P_{1}$ is maximally uniruled by $\mathbb{P}^{n-1}$ 's and there is a second chain terminating with $\alpha_{2}$, so $E_{n} / P_{1}$ is also maximally uniruled by $\mathbb{P}^{4}$ 's. (The unirulings by the $\mathbb{P}^{4}$ 's are maximal in the sense that none of the $\mathbb{P}^{4}$ 's of the uniruling are contained in any $\mathbb{P}^{5}$ on $E_{n} / P_{1}$.) The varieties parametrizing these rulings are respectively $E_{n} / P_{2}$ and $E_{n} / P_{n}$.
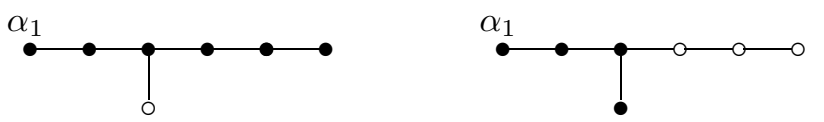

Now we address the case of exposed short roots. First note that if $X=G / P_{\alpha}=$ $B_{n} / P_{n}, G_{2} / P_{1}$ or $C_{n} / P_{1}$, then the space of $\mathbb{P}^{k}$ 's on $X$ is $\tilde{G}$-homogeneous, where $\mathcal{D}(G)$ is the fold of $\mathcal{D}(\tilde{G})$, as in these cases $G / P \simeq \tilde{G} / \tilde{P}$. In general, we have:

Theorem 4.14. If $\alpha \in S$ is an exposed short root, then for all $k, F_{k}^{\alpha}(X)$ consists of a finite number of $G$-orbits (at least two).

If $\alpha \in S$ is an exposed short root, $F_{k}^{\alpha}(X)$ can be deduced from $F_{k}\left(G / P_{\alpha}\right)$. In each of these cases we determine the unextendable linear spaces through a point explicitly. By further calculation, one can deduce all linear spaces through a point and prove the theorem.

\subsection{Natural embeddings of linear spaces}

For $X=G / P_{S} \subset \mathbb{P} V$, we have $F_{k-1}(X) \subset G(k, V) \subset \mathbb{P} \Lambda^{k} V$. Thus the connected components of the $F_{k-1}\left(G / P_{S}\right)$ 's come naturally embedded in some irreducible component of $\Lambda^{k} V$ with highest weight $\lambda$ supported on the weights dual to the roots appearing in the (unique) closed orbit $G / P_{S^{\prime}}$ consisting of $G$-homogeneous $\mathbb{P}^{k}$ 's in the component (i.e. $\mathbb{P}^{k}$ 's that are $L$-varieties in the sense of Tits). While $S^{\prime}$ can be determined pictorially, the multiplicities of the weights in general cannot. 
We now determine the multiplicities in several cases, in particular the elementary representations defined below.

Fix an end of the Dynkin diagram of $G$, and label the end node $\alpha_{1}$. Following [7], define the branch of $\alpha_{1}, \mathcal{B}\left(\alpha_{1}\right)$, as the largest chain in $\mathcal{D}(G)$ containing $\alpha_{1}$ that is isomorphic to $\mathcal{D}\left(A_{p}\right)$ or $\mathcal{D}\left(C_{p}\right)$, such that no node in $\mathcal{B}\left(\alpha_{1}\right)$ before the last has valence three. We say such a branch has length $p$. We label the roots on $\mathcal{B}\left(\alpha_{1}\right)$ as $\alpha_{1}, \ldots, \alpha_{p}$ and denote the fundamental representation corresponding to $\omega_{1}$ by $V=V_{\text {end }}=V_{\omega_{1}}$. Such an irreducible representation is called an elementary representation in [7].

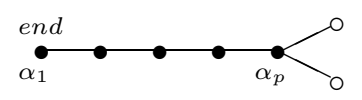

The following result is evidently due to Cartan, a proof can be found in [7] except for the 'moreover' assertions which may be verified on a case by case basis.

Proposition 4.15. With the notations above, $V_{\omega_{k}}$ is an irreducible component of $\Lambda^{k} V$, with multiplicity one for $2 \leq k \leq p$. More precisely, $\omega_{k}$ is the unique extremal weight of $\Lambda^{k} V$.

Moreover, $\Lambda^{p+1} V$ also has a unique extremal weight which is

1. $2 \omega_{p+1}$ for a double edge with arrow pointing away from $\omega_{1}$.

2. $3 \omega_{p+1}$ for a triple edge with arrow pointing away from $\omega_{1}$.

3. $\omega_{p+1}+\omega_{p+2}$ if $\omega_{p}$ corresponds to a node of valence three.

Idea of proof. One simply checks that among the weights of $V$, there is a maximal chain $\mu_{1}, \ldots, \mu_{p+1}$ with $\mu_{i}=\omega_{1}-\left(\alpha_{1}+\cdots+\alpha_{i-1}\right)$. In particular, $\mu_{1}+\cdots+\mu_{k}$ is the unique maximal weight of $\Lambda^{k} V$ for $1 \leq k \leq p+1$, and it is straightforward to check that this weight is as announced in the proposition.

It is an easy exercise to prove that the wedge product of the weight vectors corresponding to the weights $\mu_{1}, \ldots, \mu_{k}$ generate a $\mathbb{P}^{k-1}$ that is contained in the closed orbit $X_{\text {end }}$. Thus the $G$-submodule of $\Lambda^{k} V$ which hosts $F_{k-1}(X)$ is precisely the fundamental representation $V_{\omega_{k}}$.

It follows for example that in the case of simply-laced groups, we can obtain all fundamental representations from the elementary ones, in a simple geometric way.

Example 4.16. For $E_{6}$ we have three elementary representations, the minimal representation $V_{\omega_{1}}$, its dual $V_{\omega_{6}}$ and the adjoint representation $V_{\omega_{2}}$.

Start with $V_{\omega_{1}}$, so $X=E_{6} / P_{1}$ is the Cayley plane. Then $\mathbb{P} V_{\omega_{3}}$ is the ambient space for $F_{1}(X), \mathbb{P} V_{\omega_{4}}$ is the ambient space for $F_{2}(X)$ and $\mathbb{P} V_{\omega_{2}}$ is the ambient space for $F_{5}(X)$.

Start with $V_{\omega_{2}}=\mathfrak{e}_{6}$, so $X=E_{6} / P_{2}$ is the adjoint variety. Then $\mathbb{P} V_{\omega_{4}}$ is the ambient space for $F_{1}(X)$ and $\mathbb{P} V_{\omega_{3}}$ is the ambient space for $F_{4}(X)$. (Note that $E_{6} / P_{1}$ is a space of spinor varieties $D_{5} / P_{5}$ on $X$.) 
A case by case analysis with $\mathrm{LiE}[20]$ leads to the following more precise result:

Proposition 4.17. Notations as above.

1. If $X_{\mathrm{end}}$ is a minuscule variety and $V$ is not symplectic or $\left(D_{n}, \omega_{n}\right) \simeq$ $\left(D_{n}, \omega_{n-1}\right)$, then for $2 \leq k \leq p, V_{\omega_{k}}=\Lambda^{k} V$. This is the case for $(G, \omega)=$ $\left(A_{n}, \omega_{1}\right),\left(B_{n}, \omega_{1}\right),\left(D_{n}, \omega_{1}\right)$ and $\left(E_{6}, \omega_{1}\right) \simeq\left(E_{6}, \omega_{6}\right)$.

2. If $X_{\text {end }}$ is a minuscule variety and $V$ has a symplectic form $\Omega$, set $\Lambda^{\langle k\rangle} V=$ $\Lambda^{k} V /\left(\Omega \wedge \Lambda^{k-2} V\right)$. Then $V_{\text {end }-k}=\Lambda^{\langle k\rangle} V$. This is the case for $(G, \omega)=\left(C_{n}, \omega_{1}\right)$ and $\left(E_{7}, \omega_{7}\right)$.

3. If $X_{\text {end }}$ is an adjoint variety, so $V=\mathfrak{g}$, let $\Lambda^{[2]} \mathfrak{g}=\operatorname{ker}[$, ], where we consider the Lie bracket as a map [, ] : $\Lambda^{2} \mathfrak{g} \rightarrow \mathfrak{g}$. The adjoint varietes corresponding to elementary representations are those of the exceptional groups: $(G, \omega)=\left(G_{2}, \omega_{2}\right),\left(F_{4}, \omega_{1}\right),\left(E_{6}, \omega_{2}\right),\left(E_{7}, \omega_{1}\right)$ and $\left(E_{8}, \omega_{8}\right)$. In each of these cases, except for $G_{2}, V_{\text {end-1 }}=\Lambda^{[2]} \mathfrak{g}$. (And afterwards there is always a double bond or node with triple valence.)

4. If $X_{\text {end }}=G_{2} / P_{1}$ then $\left.V_{\omega_{2}}=\Lambda^{2} V_{\omega_{1}} /\left(V_{\omega_{1}}^{*}\right\lrcorner \phi\right)$ where $\phi \in \Lambda^{3} V_{\omega_{1}}$ is the defining three form.

5. If $X_{\text {end }}=F_{4} / P_{4}$, then $V_{\omega_{2}}=\Lambda^{2} V_{\omega_{4}} / \mathfrak{f}_{4}$.

Remark 4.18. Consider the case of $A_{n}$. The adjoint variety $X=G / P_{1, n}$ is the flag variety of lines in hyperplanes in $\mathbb{P}^{n}$. The space of lines in $X$ is disconnected: it is the disjoint union of $\mathbb{F}_{2, n}$ and $\mathbb{F}_{1, n-1}$, the corresponding embeddings of which are not their minimal ones, since

$$
\Lambda^{[2]} \mathfrak{s l}_{n+1}=V_{2 \omega_{1}+\omega_{n-1}} \oplus V_{\omega_{2}+2 \omega_{n}} .
$$

Remark 4.19. For $X=F_{4} / P_{4}, F_{5}(X)=F_{4} / P_{1}$, but here the variety occurs in its third Veronese re-embedding, i.e. $V_{3 \omega_{1}} \subset \Lambda^{3} V_{\omega_{4}}$. For $X=G_{2} / P_{1}, F_{2}(X)$ occurs in its second Veronese embedding, $\mathbb{P} V_{2 \omega_{1}}$.

\subsection{Dynkin diagrams via second fundamental forms}

We describe how to recover $\mathcal{D}^{*}(G)$, the marked Dynkin diagram of $G$, from the second fundamental form at a point of any $X=G / P$ where $P$ is maximal and not short:

Fix $x \in X$ and start with a marked node, which corresponds to $P$. Say $Y_{1}$ is the Segre product of Veronese re-embeddings of $k$ minuscule varieties. Then attach $k$ edges to the node, with nodes at the end of each edge. For each factor in the Segre that is minimally embedded, the edge is simple. If there is a factor that is a quadratic (resp. cubic) Veronese, then make the corresponding edge a double (resp. triple) bond. Now compute Base $\left|\mathbb{F}_{X, x}^{2}\right|$ of each factor and repeat the process starting with the node corresponding to the factor. Continue until arriving at the empty set. The resulting diagram is $\mathcal{D}(G)$. 
A shortcut: if at any point one obtains an $H / Q$ as a factor where the marked Dynkin diagram associated to $H / Q$ is known, one can simply attach the diagram. In particular, if one arrives at a $\mathbb{P}^{l}$, just attach a copy of $\mathcal{D}\left(A_{l}\right)$.

Example 4.20. Beginning with $X=E_{n} / P_{1}$, one has

$$
\begin{array}{ll}
\text { Base }\left|\mathbb{F}_{X}^{2}\right|=\mathbb{S}_{n-1}, & \text { Base }\left|\mathbb{F F}_{\mathbb{S}_{n-1}}^{2}\right|=G(2, n-1), \\
\text { Base }\left|\mathbb{F}_{G(2, n-1)}^{2}\right|=\operatorname{Seg}\left(\mathbb{P}^{1} \times \mathbb{P}^{n-2}\right), & \text { Base }\left|\mathbb{F F}_{S e g\left(\mathbb{P}^{1} \times \mathbb{P}^{n-2}\right)}^{2}\right|=\mathbb{P}^{0} \sqcup \mathbb{P}^{n-3} .
\end{array}
$$

So the construction is:

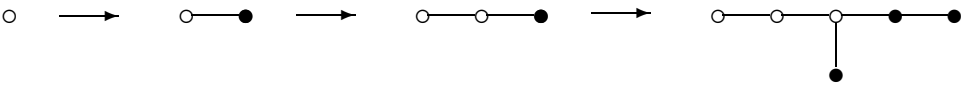

\section{Classical homogeneous varieties}

In this section we present the higher normal spaces of the classical homogeneous varieties which are not minuscule, as well as the base loci of the higher fundamental forms. We give most results without their proofs, which are computational.

\subsection{Orthogonal Grassmannians}

Let $G_{o}(k, n)$ denote the orthogonal Grassmannian of null $k$-planes in $V=\mathbb{C}^{n}$ where $V$ is equipped with a nondegenerate quadratic form $Q$ and $2 k<n$. It is a subvariety of the ordinary Grassmannian, and its minimal embedding is the Plücker embedding in $\mathbb{P} V_{\omega_{k}}=\mathbb{P}\left(\Lambda^{k} V\right)$.

Introduce the notation

$$
\left(\Lambda^{p} E^{*} \otimes \Lambda^{p} E^{*}\right)_{+}=\left\{\begin{array}{l}
S^{2}\left(\Lambda^{p} E^{*}\right) \text { if } p \text { is even } \\
\Lambda^{2}\left(\Lambda^{p} E^{*}\right) \text { if } p \text { is odd }
\end{array}\right.
$$

and

$$
\left(\Lambda^{p} E^{*} \otimes \Lambda^{p} E^{*}\right)_{-}=\left\{\begin{array}{l}
\Lambda^{2}\left(\Lambda^{p} E^{*}\right) \text { if } p \text { is even } \\
S^{2}\left(\Lambda^{p} E^{*}\right) \text { if } p \text { is odd }
\end{array}\right.
$$

Proposition 5.1. Let $E^{k}$ be the tautological vector subbundle on $G_{o}(k, n)$, let $E^{\perp} \supset E$ denote its $Q$-orthogonal complement, and let $U^{n-2 k}=E^{\perp} / E$. Then the tangent space and normal spaces of $G_{o}(k, n)$, as $H=S L(E) \times S O(U)$ modules, are

$$
\begin{gathered}
T_{1}=E^{*} \otimes U, \quad T_{2}=\Lambda^{2} E^{*} \\
N_{2}=\left(\Lambda^{2} E^{*} \otimes \Lambda^{2} U \oplus S^{2} E^{*}\right) \oplus\left(\Lambda^{2} E^{*} \otimes E^{*} \otimes U\right) \oplus\left(\Lambda^{4} E^{*} \oplus S_{22} E^{*}\right) . \\
N_{p}=\bigoplus_{a>0}\left(\Lambda^{p-a} E^{*} \otimes \Lambda^{p} E^{*} \otimes \Lambda^{a} U\right) \oplus \\
\oplus\left(\Lambda^{p} E^{*} \otimes \Lambda^{p} E^{*}\right)_{+} \oplus\left(\Lambda^{p-1} E^{*} \otimes \Lambda^{p-1} E^{*}\right)_{-} .
\end{gathered}
$$


In particular, the length of the normal graduation is $k$ when $k$ is even and the last non zero term is $N_{k} \simeq \Lambda^{k} E^{*} \otimes\left(\Lambda^{0} E^{*} \otimes \Lambda^{k} U+\cdots+\Lambda^{k} E^{*} \otimes \Lambda^{0} U\right) \oplus S^{2}\left(\Lambda^{k} E^{*}\right)$. When $k$ is odd, the length is $k+1$ and the last non zero term is $N_{k+1} \simeq \mathbb{C}$.

Remark 5.2. Note that in contrast to the case of minuscule varieties, here $N_{3}^{*} \neq$ $N_{2}^{*(1)}$.

Corollary 5.3. The base locus Base $\left|\mathbb{F F}_{G_{o}(k, n), E}^{p}\right|$ of the $p$-th fundamental form is, for $p$ even,

$$
\mathbb{P} \overline{\left\{e_{1} \otimes u_{1}+\cdots+e_{p} \otimes u_{p} \oplus e_{1} \wedge e_{2}+\cdots+e_{p-1} \wedge e_{p}, \mid e_{j} \in E^{*}, u_{j} \in U\right\}},
$$

and for $p$ odd,

$$
\mathbb{P} \overline{\left\{e_{1} \otimes u_{1}+\cdots+e_{p} \otimes u_{p} \oplus e_{1} \wedge e_{2}+\cdots+e_{p-2} \wedge e_{p-1}, \mid e_{j} \in E^{*}, \quad u_{j} \in U\right\}} .
$$

\subsection{Symplectic Grassmannians}

We let $G_{\omega}(k, 2 n)=C_{n} / P_{k}$ denote the Grassmanian of $k$-planes isotropic for a symplectic form. Its minimal embedding is to $V_{\omega_{k}}=\Lambda^{\langle k\rangle} V=\Lambda^{k} V /\left(\Omega \wedge \Lambda^{k-2} V\right)$, the $k$-th reduced exterior power of $V=\mathbb{C}^{2 n}$, where $\Omega$ denotes the symplectic form.

A straightforward computation shows that $V_{\omega_{k}}$ has the following decomposition as an $H=S L_{k} \times S p_{2 n-2 k}$-module:

$$
\Lambda^{\langle k\rangle} V=\bigoplus_{a, b} \Lambda^{b} E^{*} \otimes \Lambda^{a+b} E^{*} \otimes \Lambda^{\langle a\rangle} U
$$

Note that $U=E^{\perp} / E$ is endowed with a symplectic form induced by the symplectic form on $V=\mathbb{C}^{2 n}$.

Proposition 5.4. Let $E$ be the tautological vector sub bundle on $G_{\omega}(k, 2 n)$, let $E^{\perp} \supset E$ denote the $\Omega$-orthogonal complement to $E$ and let $U=E^{\perp} / E$. Then the tangent space and normal spaces of $G_{\omega}(k, 2 n)$ are, as $H$-modules,

$$
\begin{gathered}
T_{1}=E^{*} \otimes U, \quad T_{2}=S^{2} E^{*}, \\
N_{2}=\Lambda^{2} E^{*} \otimes \Lambda^{\langle 2\rangle} U \oplus S_{21} E^{*} \otimes U \oplus S_{22} E^{*}, \\
N_{p}=\bigoplus_{a+b+c=p} \Lambda^{\langle a\rangle} U \otimes S_{\underbrace{2 \ldots 2}_{b-c}} \underbrace{1 \ldots 1}_{a+2 c} E^{*} \\
=\bigoplus_{d+e=p} \Lambda^{d} U \otimes S_{e}^{\underbrace{}_{d} \ldots 2} \underbrace{1 \ldots 1}_{d} E^{*} .
\end{gathered}
$$

In particular, the length of the normal graduation is equal to $k$, the last non zero term being $N_{k} \simeq \Lambda^{k}(\mathbb{C} \oplus U)$. 


\section{Corollary 5.5.}

$$
\text { Base }\left|\mathbb{F}_{G_{\omega}(k, 2 n), E}^{2}\right|=\overline{\mathbb{P}\left\{e \otimes u \oplus e^{2} \mid e \in E^{*} \backslash\{0\}, u \in U \backslash\{0\}\right\}} .
$$

This base locus contains an open and dense P-orbit, the boundary of which is the union of the two (disjoint) closed $\mathrm{H}$-orbits

$$
Y_{1} \simeq \mathbb{P}^{k-1} \times \mathbb{P}^{2 n-2 k-1} \subset \mathbb{P}\left(T_{1}\right) \quad \text { and } \quad Y_{2} \simeq v_{2}\left(\mathbb{P}^{k-1}\right) \subset \mathbb{P}\left(T_{2}\right)
$$

Proof. This can be seen directly. A line in $G_{\omega}(k, 2 n)$ through a point $E$ is given by a $(k-1)$-plane $H \subset E$, and a $(k+1)$-plane $K \supset E . K$ does not need to be isotropic, each point of the corresponding line is generated by $H$ and a vector of $K$, and is isotropic if and only if this vector is $\omega$-orthogonal to $H$. The condition on $K$ is thus $K \subset H^{\perp} . K$ is therefore determined by a line in $H^{\perp} / E \simeq U \oplus H^{\perp} / E^{\perp}$.

If $e \in E^{*}$ is an equation of $H$, the line $H^{\perp} / E^{\perp} \simeq(E / H)^{*} \subset E^{*}$ is generated by $E$, so that a vector in $H^{\perp} / E$ can be written as $u \oplus \lambda e$, where $u \in E$. Our claim follows, the closed orbits $Y_{1}$ and $Y_{2}$ corresponding to the cases where $u$ or $\lambda$ is equal to zero.

Corollary 5.6. More generally, the base locus of the $p$-th fundamental form is Base $\left|\mathbb{F F}_{G_{\omega}(k, 2 n), E}^{p}\right|=\mathbb{P} \overline{\left\{e_{1} \otimes u_{1}+\cdots+e_{p} \otimes u_{p} \oplus e_{1}^{2}+\cdots+e_{p}^{2}, \mid e_{j} \in E^{*}, u_{j} \in U\right\}}$.

Remark 5.7. Since $C_{n} / P_{k}=G_{\omega}(k, 2 n)$ is a subvariety of the ordinary Grassmannian $G(k, 2 n)$, the $\mathbb{P}^{l}$ 's it contains are easy to describe: letting $M_{0}^{a}, N_{0}^{b}$ denote fixed linear spaces of dimensions $a$ and $b$, they are of the form

$$
\left\{M_{0}^{k-1} \subset L \subset N_{0}^{k+l} \subset M_{0}^{\perp}\right\},
$$

with $l \leq 2 n-2 k+1$, or

$$
\left\{M_{0}^{k-l} \subset L \subset N_{0}^{k+1} \subset M_{0}^{\perp}\right\}
$$

with $1<l \leq k$ and $N_{0}$ isotropic. This second family of $\mathbb{P}^{l}$ 's has two $C_{n}$-orbits, while the first family breaks into a number of $C_{n}$-orbits that grows with $k$, indexed by the rank of the restriction of $\Omega$ to $N_{0}$.

\subsection{Odd spinor varieties}

We call the homogeneous spaces $B_{n} / P_{n}$ the odd spinor varieties. They are the usual $D_{n}$-spinor varieties seen as $B_{n}$-homogeneous spaces.

Proposition 5.8. The tangent space and normal spaces of the odd spinor varieties $B_{n} / P_{n}$, as $H=A_{n-1}$-modules, are ( $\left.\operatorname{dim} E=n\right)$ :

$$
\begin{aligned}
T_{1} & =E^{*}, \quad T_{2}=\Lambda^{2} E^{*}, \\
N_{p} & =\Lambda^{2 p-1} E^{*} \oplus \Lambda^{2 p} E^{*} .
\end{aligned}
$$




\section{Corollary 5.9.}

$$
\text { Base }\left|\mathbb{F F}_{B_{n} / P_{n}}^{2}\right|=\mathbb{P} \overline{\left\{e \oplus e \wedge f, e \in E^{*} \backslash\{0\}, f \in E^{*} \backslash \mathbb{C} e\right\}} \simeq G(2, n) .
$$

This base locus contains an open and dense H-orbit, the boundary of which is the union of the two (disjoint) closed $\mathrm{H}$-orbits

$$
Y_{1}=\mathbb{P} E^{*}=\mathbb{P}\left(T_{1}\right) \quad \text { and } \quad Y_{2} \simeq G\left(2, E^{*}\right)=G(2, n-1) \subset \mathbb{P}\left(T_{2}\right) .
$$

The base locus of the $k$-th fundamental form is

$$
\text { Base }\left|\mathbb{F F}_{B_{n} / P_{n}}^{k}\right|=\mathbb{P} \overline{\left\{e \oplus \Omega, e \in E^{*} \backslash\{0\}, \operatorname{rank} \Omega_{\mid e^{\perp}}<\operatorname{rank} \Omega \leq 2 k-2\right\}} .
$$

Proof. Consider $V^{2 n+1} \subset W^{2 n+2}$, and $E^{n} \subset F^{n+1}$, where $F$ is a null plane in $W$. let $L=E^{\perp} \subset F$ Then $\Lambda^{\bullet} E^{*} \simeq \Lambda^{\text {even }} F^{*}$. We may write $N_{2}=\Lambda^{3} E^{*} \otimes L \oplus \Lambda^{4} E^{*}=$ $\Lambda^{4} F=I_{2}(G(2, F))$. The analogous identities hold for the higher normal spaces. After all, this is the same projective variety as $D_{n} / P_{n}$.

Remark 5.10. The varieties parametrizing the $\mathbb{P}^{l}$ 's of $B_{n} / P_{n}=D_{n+1} / P_{n+1}$ are stratified as $B_{n}$-spaces. For $k \geq 2$, a connected component of this variety is $D_{n+1} / P_{n, n-k-1}$. If $\tilde{V}=\mathbb{C}^{2 n+2}$ is endowed with a nondegenerate quadratic form, a point of this space is a flag $F_{0} \subset M_{0}$ of isotropic subspaces of $\tilde{V}$ of respective dimensions $n-k-1$ and $n$. The associated line in $D_{n+1} / P_{n+1}$ is the space of $n$-dimensional isotropic subspaces of $\tilde{V}$ containing $F_{0}$ and cutting $M_{0}$ in dimension $n-1$. Let $V$ be the hyperplane of $\tilde{V}$ preserved by $B_{n}$. Then the $B_{n}$-orbits inside $D_{n+1} / P_{n, n-k-1}$ are indexed by the relative position of $F_{0}$ and $V$. There is a closed orbit corresponding to $F_{0} \subset V$, isomorphic to $B_{n} / P_{n-k-1}$, and its complement is an open orbit. For $k=1$ or $k=3$, there is another connected component, parametrized by $D_{n+1} / P_{n-1}$ and $D_{n+1} / P_{n-2}$ respectively. Each of them has two $B_{n}$-orbits, the closed orbits being $B_{n} / P_{n-1}$ and $B_{n} / P_{n-2}$ respectively.

\section{Exceptional short roots and the octonions}

In this section we calculate Base $\left|\mathbb{F F}_{X, x}^{2}\right|$ for the exceptional spaces corresponding to short roots, and give geometric interpretations of these varieties and their linear spaces in terms of the octonions.

\section{1. $G_{2} / P_{1}$}

As an algebraic variety, $G_{2} / P_{1}$ is a familiar space, $G_{2} / P_{1}=\mathbb{Q}^{5} \subset \mathbb{P}^{6}$. Studying it from an octonionic perspective will help us to understand $F_{4} / P_{4}$ by analogy.

Identify $\mathbb{C}^{7} \simeq \operatorname{Im} \mathbb{O}=V_{\omega_{1}}=V$. Let $\phi \in \Lambda^{3} V^{*}$ be a generic element and let $\rho$ : $G L(V) \rightarrow G L\left(\Lambda^{3} V^{*}\right)$ be the induced representation. Here are some descriptions 
of $G_{2} \subset G L(V)$ (see [10] pp. 114, 116, 278 and [23] chapter 2):

$$
\begin{aligned}
G_{2} & =\operatorname{Aut}(\mathbb{O}) \\
& =\{g \in G L(V) \mid \rho(g) \phi=\phi\} \\
& =\left\{g=\left(g_{+}, g_{-}, g_{0}\right) \in \operatorname{Spin}_{8}(V) \mid g_{+}=g_{-}=g_{0}\right\} .
\end{aligned}
$$

The third line should be understood as follows: let $S_{+}, S_{-}, V_{0}$ denote the vector and two spin representations of $\operatorname{Spin}_{8}$ and choose appropriately an identification of each of the three spaces with $\mathbb{O}$, so that they are acted on by $g \in \operatorname{Spin}_{8}$ in three different ways, call them $\left(g_{+}, g_{-}, g_{0}\right)$. In this case (see [10] p. 278), the triality principle of E. Cartan leads to the identification

$$
\operatorname{Spin}_{8}=\left\{\left(g_{+}, g_{-}, g_{0}\right) \in S O(\mathbb{O}) \times S O(\mathbb{O}) \times S O(\mathbb{O}) \mid g_{+}(u v)=g_{-}(u) g_{0}(v)\right\},
$$

where $u v$ denotes octonionic multiplication. When the three coincide one obtains an automorphism of the octonions, showing the equivalence of the second and third definition. Harvey's description is explicit in bases. The connection between the first two interpretations is that if one makes suitable identifications, for $u, v, w \in$ $\operatorname{Im} \mathbb{O}$, we have $\phi(u, v, w)=\operatorname{Re}[(u v) w]$.

The first definition is due to R. Bryant. It shows that $G_{2}$ is not really an exceptional group, because it is defined by a generic form. (Generic three forms on $\mathbb{C}^{m}$ for $m>8$ are not preserved by a positive dimensional group. For $m=6,8$, the groups preserving such a form are classical.)

The third interpretation can be understood in terms of folding Dynkin diagrams:

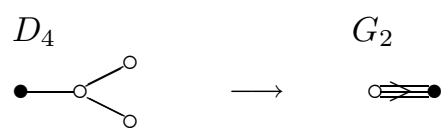

This indicates that $G_{2} / P_{1}$ should be be understandable in terms of $D_{4} / P_{1}=\mathbb{Q}^{6}$, and in fact it is a generic hyperplane section. $\operatorname{Im} \mathbb{O} \subset \mathbb{O}$ should be thought of as the traceless elements, where the trace of an element is its "real" part and we call the hyperplane section $\{\operatorname{tr}=0\}$. In what follows, $u v$ etc... refers to octonionic multiplication.

Proposition 6.1. Consider $\mathbb{Q}^{5} \simeq G_{2} / P_{1}=\mathbb{P}(\operatorname{Im} \mathbb{O})_{0} \subset \mathbb{P}(\operatorname{Im} \mathbb{O}) \simeq \mathbb{P}\left(V_{\omega_{1}}\right)$.

Then $T_{x} G_{2} / P_{1}=T_{1} \oplus T_{2} \oplus T_{3}$ as an $H=S L_{2}$-module. Let $A=\mathbb{C}^{2}$, the standard representation of $S L_{2}$. Then $T_{1}=A, T_{2}=\mathbb{C}$ (the trivial representation) and $T_{3}=A^{*}$. Moreover, in a suitable normalization,

$$
\text { Base }\left|\mathbb{F F}_{G_{2} / P_{1}}^{2}\right|=\mathbb{P}\left\{a \oplus t \oplus a^{*} \mid\left\langle a, a^{*}\right\rangle=t^{2}\right\} \text {. }
$$

Proposition 6.2. We have the following octonionic interpretations:

$$
\begin{aligned}
G_{2} / P_{1} & =\left\{[u] \in \mathbb{P}(\operatorname{Im} \mathbb{O}) \mid u^{2}=0\right\} \\
\hat{T}_{[u]} G_{2} / P_{1} & =\{v \in \operatorname{Im} \mathbb{O} \mid u v+v u=0\}=\{v \in \operatorname{Im} \mathbb{O} \mid \operatorname{Re}(u v)=0\} \\
\hat{T}_{[u] 1} G_{2} / P_{1} & =\{v \in \operatorname{Im} \mathbb{O} \mid u v=0\} .
\end{aligned}
$$


Proposition 6.3. The space $\mathbb{G}\left(\mathbb{P}^{1}, G_{2} / P_{1}\right)$ of lines on $G_{2} / P_{1}$, has the following description:

$$
\mathbb{G}\left(\mathbb{P}^{1}, G_{2} / P_{1}\right)=\left\{\mathbb{P}\{u, v\} \mid[u],[v] \in G_{2} / P_{1} \text { such that } u v+v u=0\right\} .
$$

Note that $\mathbb{G}\left(\mathbb{P}^{1}, G_{2} / P_{1}\right)=G_{o}(2,7)$ and in particular is of dimension seven.

The space $G_{2} / P_{2}=\mathbb{G}_{0}\left(\mathbb{P}^{1}, \mathbb{P}(\operatorname{Im} \mathbb{O})_{0}\right)$ of $G_{2}$-homogeneous lines on $G_{2} / P_{1}$ has dimension 5 , and admits the following descriptions:

i. $\left.G_{2} / P_{2}=\left\{\mathbb{P} E \in \mathbb{G}\left(\mathbb{P}^{1}, \mathbb{P} V_{\omega_{1}}\right) \mid E\right\lrcorner \phi=0\right\}$

ii. $G_{2} / P_{2}=\left\{\mathbb{P} E=\mathbb{P}\{u, v\} \mid[u],[v] \in G_{2} / P_{1}, u v=0\right\}$.

Proofs are left to the reader. The arguments are similar to, but simpler than the arguments for the $F_{4} / P_{4}$ case below.

Remark 6.4. Recall that $V_{\omega_{2}}$ is the adjoint representation of $G_{2}$, so that $G_{2} / P_{2}$ is an adjoint variety. The relation with our description of $G_{2} / P_{2}$ as a space of special lines on the quadric $G_{2} / P_{1}$ is as follows. Let $[u],[v] \in G_{2} / P_{1}$ be such that $u v=v u=0$. One can then check that the map

$$
d_{u, v}(z)=u(v z)-v(u z), \quad z \in \mathbb{O},
$$

defines a nilpotent derivation of $\mathbb{O}$, with $d_{u, v}^{2}=0$.

Note that $G_{Q}(3,7) \simeq Q^{6}$, the space of $\mathbb{P}^{2}$ 's on $G_{2} / P_{1}$, contains a special family of planes isomorphic to $G_{2} / P_{1}$ as follows: through each point of $G_{2} / P_{1}$ there is a plane contained in $G_{2} / P_{1}$ tangent to $T_{1}$ (and it is completely tangent to $T_{1}$ at this point only). Perhaps it is better to say the space of special $\mathbb{P}^{2}$ 's is parametrized by $v_{2}\left(G_{2} / P_{1}\right)$ as the variety sits inside $V_{2 \omega_{1}} \subset \Lambda^{3} V_{\omega_{1}}$. (Although $V_{\omega_{1}} \subset \Lambda^{3} V_{\omega_{1}}$, the Veronese re-embedding is the correct factor as were there linear spaces on the parameter space, they would determine larger linear spaces on $G_{2} / P_{1}$ which do not exist.)

\subsection{The Cayley plane $\mathbb{O P}^{2}$}

Let $\mathcal{J}_{3}(\mathbb{O})$ be the space of $3 \times 3 \mathbb{O}$-Hermitian symmetric matrices

$$
\mathcal{J}_{3}(\mathbb{O})=\left\{A=\left(\begin{array}{lll}
r_{1} & \overline{x_{3}} & \overline{x_{2}} \\
x_{3} & r_{2} & \overline{x_{1}} \\
x_{2} & x_{1} & r_{3}
\end{array}\right), r_{i} \in \mathbb{C}, x_{j} \in \mathbb{O}\right\} .
$$

$\mathcal{J}_{3}(\mathbb{O})$ can be equipped with the structure of a Jordan algebra for the commutative product $A \circ B=\frac{1}{2}(A B+B A)$, where $A B$ is the usual matrix product. $\operatorname{dim}_{\mathbb{C}} \mathcal{J}_{3}(\mathbb{O})=27$ and it is a model for the $E_{6}$-module $V_{\omega_{1}}$. There is a well-defined determinant on $\mathcal{J}_{3}(\mathbb{O})$, which is defined by same expression as the classical determinant in terms of traces:

$$
\operatorname{det} A=\frac{1}{6}(\operatorname{trace} A)^{3}-\frac{1}{2}(\operatorname{trace} A)\left(\operatorname{trace} A^{2}\right)+\frac{1}{3} \operatorname{trace} A^{3} .
$$


$E_{6}$ is the subgroup of $G L\left(\mathcal{J}_{3}(\mathbb{O})\right)=G L(27, \mathbb{C})$ preserving det. The notion of rank one matrices is also well defined and the Cayley plane, $E_{6} / P_{1}=\mathbb{O P}^{2} \subset \mathbb{P}\left(\mathcal{J}_{3}(\mathbb{O})\right)$ is the projectivization of the rank one elements, with ideal the $2 \times 2$ minors (see $[13])$.

Since $\alpha_{1}$ is not short, all linear spaces on $\mathbb{O P}^{2}$ are described by Tits geometries. In particular, $E_{6} / P_{3}$ is the space of lines on $\mathbb{O P}^{2}$ and $E_{6} / P_{2}$ is the space of $\mathbb{P}^{5}$ 's on $\mathbb{O P}^{2}$.

6.3. $F_{4} / P_{4}=\mathbb{O P}_{0}^{2}$

Here are some descriptions of $F_{4} \subset G L\left(\mathcal{J}_{3}(\mathbb{O})\right)$ :

$$
\begin{aligned}
F_{4} & =\left\{g \in G L\left(\mathcal{J}_{3}(\mathbb{O})\right) \mid \operatorname{tr}\left((\rho(g) A)^{i}\right)=\operatorname{tr} A^{i} \text { for } i=1,2,3\right\} \\
& =\operatorname{Aut}\left(\mathcal{J}_{3}(\mathbb{O})\right) \\
& =\left\{g \in E_{6} \mid g_{+}=g_{-}\right\}
\end{aligned}
$$

The third description is motivated by folding of Dynkin diagrams:

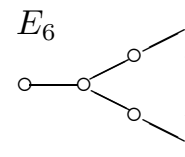

$F_{4}$

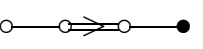

The equivalence of the second and third descriptions can be proved by using the quadratic form $\operatorname{tr}\left(A^{2}\right)$ to identify $\mathcal{J}_{3}(\mathbb{O})$ with $\mathcal{J}_{3}(\mathbb{O})^{*}$ and considering $g_{+}$(resp. $\left.g_{-}\right)$as the two resulting elements of $G L\left(\mathcal{J}_{3}(\mathbb{O})\right)$. Harvey shows that the second definition implies the first [10] p. 296. For the first definition, one only needs two of the three forms to be preserved, as any group preserving two preserves the third.

Geometric folding indicates $F_{4} / P_{4}$ should be understood in terms of $\mathbb{O P}^{2}$, and, as with $G_{2} / P_{1}$ above, it is the hyperplane section $\{\operatorname{tr}=0\}$. In what follows, $A B$ denotes the usual matrix product of $A$ and $B$. Note that $A^{2}=A \circ A$.

Proposition 6.5. Consider $\mathbb{O P}_{0}^{2}=F_{4} / P_{4} \subset \mathbb{P}\left(\mathcal{J}_{3}(\mathbb{O})_{0}\right) \simeq \mathbb{P}\left(V_{\omega_{4}}\right)$. Then $T_{x}\left(F_{4} / P_{4}\right)=T_{1} \oplus T_{2}$ as an $H=\operatorname{Spin}_{7}$-module. Let $U$ be the 7-dimensional vector representation of $\operatorname{Spin}_{7}$ and $\mathcal{S}(U)$ the spin representation, then $T_{1}=\mathcal{S}(U)$ and $T_{2}=U$. The spinor variety $Y_{1}=\mathbb{S}(U)$ is a six dimensional quadric, and $Y_{2}=\mathbb{Q}^{5}$ is a five dimensional quadric. Moreover, we may identify $T_{1} \simeq \mathbb{O}$ and $T_{2} \simeq \operatorname{Im} \mathbb{O}$ and with this identification

$$
\text { Base }\left|\mathbb{F F}_{\mathbb{O P}_{0}^{2}}^{2}\right|=\mathbb{P} \overline{\left\{(u, v) \in T_{1} \oplus T_{2} \mid u \bar{u}=0, v \bar{v}=0, u \bar{v}=0\right\}}=\mathbb{S}_{5} \cap H
$$

where $\mathbb{S}_{5} \cap H$ is a generic hyperplane section of the spinor variety $\mathbb{S}_{5}=D_{5} / P_{5}$. In particular, Base $\left|\mathbb{F F}_{\mathbb{O P}_{0}^{2}}^{2}\right|$ is of dimension 9 , and is the closure of a $\mathrm{Spin}_{7}$-orbit, the boundary of which is the disjoint union of $Y_{1}$ and $Y_{2}$. It is not homogeneous for any group. 
Proof. The decomposition of the tangent space follows from $§ 2.3$. Moreover, Base $\left|\mathbb{F F}_{\mathbb{O} \mathbb{P}_{0}^{2}}^{2}\right|$ must be a generic hyperplane section of $\mathbb{S}_{5}=$ Base $\left|\mathbb{F F}_{\mathbb{O} \mathbb{P}^{2}}^{2}\right|$ because $F_{4} / P_{4}$ is a generic hyperplane section of $E_{6} / P_{6}$. The explicit description follows using the description of $T_{x} \mathbb{O P}^{2} \simeq \mathbb{O} \oplus \mathbb{O}$ and the explicit description of $I I_{\mathbb{O P}^{2}}$ in [13], verifying that $\mathbb{O} \oplus \mathbb{O}_{0}$ is indeed a generic hyperplane section or using the explicit description below. Finally, we check that there is no homogeneous space of dimension 9 homogeneously embedded in a $\mathbb{P}^{14}$.

Proposition 6.6. We have the following octonionic interpretations:

$$
\begin{aligned}
\mathbb{O P}_{0}^{2}=F_{4} / P_{4} & =\left\{[A] \in \mathbb{P} \mathcal{J}_{3}(\mathbb{O})_{0} \mid A^{2}=0\right\} \\
\hat{T}_{[A]} \mathbb{O P}_{0}^{2} & =\left\{B \in \mathcal{J}_{3}(\mathbb{O})_{0} \mid A \circ B=0\right\} \\
\hat{T}_{1[A]} \mathbb{O P}_{0}^{2} & =\left\{B \in \mathcal{J}_{3}(\mathbb{O})_{0} \mid A B=0\right\}
\end{aligned}
$$

Proof. A calculation shows that an element $A \in \mathcal{J}_{3}(\mathbb{O})$ is rank one and traceless if and only if $A^{2}=0$. Differentiation yields the second line.

To prove the third line, we first need to show that if $[A] \in \mathbb{O P}_{0}^{2}$ and $B \in$ $\hat{T}_{[A]} \mathbb{O P}_{0}^{2}$, the equation $A B=0$ is $F_{4}$ invariant (although the matrix product $A B$ is not $F_{4}$ invariant). Note that $F_{4}$ is generated by $S_{3}$ and $\mathrm{Spin}_{8}$, where the action of $g \in S O_{3}$ is by $A \mapsto g A^{t} g$, and that of $\left(g_{+}, g_{-}, g_{0}\right) \in \operatorname{Spin}_{8}$ by

$$
\left(\begin{array}{lll}
r_{1} & \overline{x_{3}} & \overline{x_{2}} \\
x_{3} & r_{2} & \overline{x_{1}} \\
x_{2} & x_{1} & r_{3}
\end{array}\right) \mapsto\left(\begin{array}{ccc}
r_{1} & \overline{g_{+}\left(x_{3}\right)} & \overline{g_{-}\left(x_{2}\right)} \\
g_{+}\left(x_{3}\right) & r_{2} & \overline{g_{0}\left(x_{1}\right)} \\
g_{-}\left(x_{2}\right) & g_{0}\left(x_{1}\right) & r_{3}
\end{array}\right) .
$$

(This defines an automorphism of the Jordan algebra $\mathcal{J}_{3}(\mathbb{O})_{0}$ because of the triality principle.) The $\mathrm{SO}_{3}$ invariance is clear. Moreover, if we take

$$
A=\left(\begin{array}{ccc}
i & 1 & 0 \\
1 & -i & 0 \\
0 & 0 & 0
\end{array}\right)
$$

then

$$
\hat{T}_{[A]} \mathbb{O P}_{0}^{2}=\left(\begin{array}{ccc}
i \operatorname{tr}\left(x_{3}\right) & \overline{x_{3}} & i \overline{x_{1}} \\
x_{3} & -i \operatorname{tr}\left(x_{3}\right) & \overline{x_{1}} \\
i x_{1} & x_{1} & 0
\end{array}\right)
$$

where $\operatorname{tr}(u)=u-u_{0}=\frac{1}{2}(u+\bar{u})$ is the "real" part of $u$. The $\operatorname{Spin}_{8}$ invariance of the equation $A B=0$ is a straightforward calculation, and follows again from the triality principle. With this model, $\left\{B \in \hat{T}_{[A]} \mathbb{O P}_{0}^{2} \mid A B=0\right\} \simeq\left\{x_{1}, \operatorname{tr}\left(x_{3}\right)\right\}$ and we may consider $\left\{x_{1}\right\} \subset \hat{T}_{[A]} /\{A\} \simeq T$. Note that $T$ is acted on by the subgroup of $\operatorname{Spin}_{8}$ that preserves $A$, which means that $g_{+}(1)=1$. By [10] p. 285,

$$
\begin{aligned}
\operatorname{Spin}_{7} & =\left\{\left(g_{+}, g_{-}, g_{0}\right) \in \operatorname{Spin}_{8} \mid g_{-}=g_{0}\right\} \\
& =\left\{\left(g_{+}, g_{-}, g_{0}\right) \in \operatorname{Spin}_{8} \mid g_{+}(1)=1 \in \mathbb{O}\right\}
\end{aligned}
$$

(Note that this embedding of $\operatorname{Spin}_{7}$ in $\operatorname{Spin}_{8}$ is not the standard one). Thus we explicitly see the $\operatorname{Spin}_{7}=H$ action on $T$ and the decomposition of $T$ into 
$T_{1} \simeq\left\{x_{1}\right\}$ and $T_{2} \simeq\left\{\left(x_{3}\right)_{0}\right\}$, respectively as the spin and vector representations. In particular, $\left\{T_{1}+A\right\}=\{B \in \hat{T} \mid A B=0\}$.

Proposition 6.7. The space $\mathbb{G}\left(\mathbb{P}^{1}, \mathbb{O P}_{0}^{2}\right)$ of lines on $\mathbb{O P}_{0}^{2}$ has dimension 23 , and admits the following description:

$$
\mathbb{G}\left(\mathbb{P}^{1}, \mathbb{O} \mathbb{P}_{0}^{2}\right)=\left\{\mathbb{P}\{A, B\} \mid[A],[B] \in \mathbb{O P}_{0}^{2} \text { such that } A \circ B=0\right\}
$$

The space $F_{4} / P_{3}=\mathbb{G}_{0}\left(\mathbb{P}^{1}, \mathbb{O P}_{0}^{2}\right)$ of $F_{4}$-homogeneous lines on $\mathbb{O P}_{0}^{2}$ has dimension 20, and admits the following description:

$$
\mathbb{G}_{0}\left(\mathbb{P}^{1}, \mathbb{O P}_{0}^{2}\right)=\left\{\mathbb{P}\{A, B\} \mid[A],[B] \in \mathbb{O P}_{0}^{2} \text { such that } A B=0\right\} .
$$

Proof. The geometric descriptions of $\mathbb{G}\left(\mathbb{P}^{1}, \mathbb{O P}_{0}^{2}\right)$ and $\mathbb{G}_{0}\left(\mathbb{P}^{1}, \mathbb{O P}_{0}^{2}\right)$ follow immediately from proposition 6.6 , because $F_{4} / P_{3}$ is the space of lines on $F_{4} / P_{4}$ tangent to $Y_{1}$. Moreover,

$$
\operatorname{dim}\left(\mathbb{G}\left(\mathbb{P}^{1}, \mathbb{O P}_{0}^{2}\right)\right)=\operatorname{dim} \mathbb{O} \mathbb{P}_{0}^{2}+\operatorname{dim}\left(\text { Base }\left|\mathbb{F}_{\mathbb{O P}_{0}^{2}}^{2}\right|\right)-1=15+9-1=23
$$

verifies the dimension assertion.

Proposition 6.8. There are four types of maximal (i.e. unextendable) linear spaces through a point of $F_{4} / P_{4}$ : $\mathbb{P} T_{2}$.

The space of $\mathbb{P}^{5}$ 's which is 5-dimensional, parametrized by the quadric $Q^{5} \subset$

A space of $\mathbb{P}^{4}$ 's which is 6-dimensional, parametrized by the quadric $Q^{6} \subset \mathbb{P} T_{1}$ or equivalently $\mathbb{S}_{Q^{5}}$, the variety of $\mathbb{P}^{2}$ 's in $Q^{5} \subset \mathbb{P} T_{2}$.

A space of $\mathbb{P}^{4}$ 's which is 6 -dimensional and having two components, the two copies of $\mathbb{S}_{Q^{6}}$, the variety of $\mathbb{P}^{3}$ 's in $Q^{6} \subset \mathbb{P} T_{1}$.

All other linear spaces can be deduced from these.

Proof. A $\mathbb{P}^{k}$ in $F_{4} / P_{4}$ corresponds to a $\mathbb{P}^{k-1}$ in $\mathbb{S}_{5} \cap H$. Let $L=\mathbb{P}^{m} \subset \mathbb{S}_{5} \cap H$. The dimension $d_{2}$ of its projection $p_{2}(L)$ onto $Q^{5} \subset \mathbb{P} T_{2}$ is $P_{4}$ invariant.

We choose a splitting $T=T_{1} \oplus T_{2}$ in order to use the equations above describing Base $\left|\mathbb{F F}_{X, x}^{2}\right|$. Relative to a choice of splitting, $L$ is just the span of $p_{1}(L)$ and $p_{2}(L)$ so we can analyze $L$ accordingly.

Since $B_{3}$ acts transitively on $G_{Q}\left(k, T_{2}\right)$, we may choose convenient $k$-planes to calculate with.

Without loss of generality, take $v=\varepsilon_{1}+i \varepsilon_{2} \in T_{2}$, Then, writing $u=\alpha_{0}+$ $\alpha_{1} \varepsilon_{1}+\cdots+\alpha_{7} \varepsilon_{7}$, and using the standard octonionic multiplication table (e.g., see [13]) the condition $u v=0$ implies $u=i \alpha_{3}+\alpha_{1} \varepsilon_{1}+i \alpha_{1} \varepsilon_{2}+\alpha_{3} \varepsilon_{3}+i \alpha_{5} \varepsilon_{4}+$ $\alpha_{5} \varepsilon_{5}+\alpha_{6} \varepsilon_{6}+i \alpha_{6} \varepsilon_{7}$. In other words, we obtain a $\mathbb{P}_{v}^{3} \subset Q^{6}$ "polar" to $v$ which gives rise to an unextendable $L^{4}=\left\langle v, \mathbb{P}_{v}^{3}\right\rangle$. Note that we automatically have $u \bar{u}=0$. Taking $M=\left\langle v, v^{\prime}\right\rangle$ with $v^{\prime}=\varepsilon_{6}+i \varepsilon_{7}$, the additional condition $u v^{\prime}=0$ implies $u=\alpha\left(\varepsilon_{6}+i \varepsilon_{7}\right)$, i.e., is a point $q \in Q^{6}$. A similar computation shows that any 
$q \in Q^{6}$ has a $\mathbb{P}^{2}$ 's worth of points in $Q^{5}$ 'polar' to it so we obtain an unextendable $L^{3}=\left\langle\mathbb{P}_{q}^{2}, q\right\rangle$. If $p_{2}(L)$ is empty, then we are of course free to take one of the two families of $\mathbb{P}^{3}$ 's on $Q^{6}$ as our maximal linear space.

6.4. $F_{4} / P_{3}=\mathbb{G}_{0}\left(\mathbb{P}^{1}, \mathbb{O P}_{0}^{2}\right)$

Proposition 6.9. Consider $F_{4} / P_{3} \subset \mathbb{P} V_{\omega_{3}}$. Then $T=T_{1} \oplus T_{2} \oplus T_{3} \oplus T_{4}$ as an $H=S L_{3} \times S L_{2}$-module. Let $\operatorname{dim} E=3$ and $\operatorname{dim} U=2$, then

$$
\begin{aligned}
& \quad T_{1}=E^{*} \otimes U, \quad T_{2}=E \otimes S^{2} U, \quad T_{3}=U, \quad T_{4}=E^{*}, \\
& \text { Base }\left|\mathbb{F F}_{\mathbb{G}_{0}\left(\mathbb{P}^{1}, \mathbb{O P}_{0}^{2}\right)}^{2}\right| \\
& =\overline{\mathbb{P}} \overline{\left\{e^{*} \otimes u+e \otimes u^{2} \in T_{1} \oplus T_{2} \mid e^{*} \in E^{*}, e \in E, u \in U,\left\langle e^{*}, e\right\rangle=0\right\} .}
\end{aligned}
$$

This base locus $B$ is a nontrivial $\mathbb{Q}^{4}$-bundle over $\mathbb{P} U=\mathbb{P}^{1}$. In particular, $\operatorname{dim} B=$ 5 and it has a dense open $S L_{3} \times S L_{2}$-orbit, the boundary of which is the union of the two closed orbits $Y_{1} \subset \mathbb{P} T_{1}$ and $Y_{2} \subset \mathbb{P} T_{2}$. It is not homogeneous for any Lie group.

Proposition 6.10. The space $\mathbb{G}\left(\mathbb{P}^{1}, F_{4} / P_{3}\right)$ of $\mathbb{P}^{1}$ 's on $F_{4} / P_{3}$ has dimension 24 , and admits the following description:

$$
\begin{aligned}
& \mathbb{G}\left(\mathbb{P}^{1}, F_{4} / P_{3}\right) \\
& =\left\{\{A\} \subset\{A, B, C\} \mid[A],[B],[C] \in \mathbb{O P}_{0}^{2} \text { such that } A B=A C=0, B \circ C=0\right\} .
\end{aligned}
$$

The space $F_{4} / P_{2,4}=\mathbb{G}_{0}\left(\mathbb{P}^{1}, \mathbb{O P}_{0}^{2}\right)$ of $F_{4}$-homogeneous $\mathbb{P}^{1}$ 's on $F_{4} / P_{3}$ has dimension 22 , and admits the following description:

$$
\begin{aligned}
F_{4} / P_{2,4} & =\mathbb{G}_{0}\left(\mathbb{P}^{1}, \mathbb{O P}_{0}^{2}\right) \\
& =\left\{\{A\} \subset\{A, B, C\} \mid[A],[B],[C] \in \mathbb{O P}_{0}^{2} \text { with } A B=A C=B C=0\right\} .
\end{aligned}
$$

Corollary 6.11. There are two types of maximal linear spaces passing through a point of $F_{4} / P_{3}$; the $\mathbb{P}^{3}$ 's corresponding to a $\mathbb{P}^{2}$ in some quadric in a fiber of Base $\left|\mathbb{F}_{\mathbb{G}_{0}\left(\mathbb{P}^{1}, \mathbb{O P}_{0}^{2}\right)}^{2}\right|$ considered as a fibration and the $\mathbb{P}^{2}$ 's corresponding to the $\mathbb{P}^{1}$ in the base.

Proof of 6.10 . We have $F_{4} / P_{3} \subset G(2,26)$ so a line on $F_{4} / P_{3}$ must be a line of the Grassmanian as well. Lines on $G(2,26)$ are determined by the choice of a flag $\mathbb{P}^{0} \subset \mathbb{P}^{2}$. Here we need $[A]=\mathbb{P}^{0} \in F_{4} / P_{4}$ in both cases.

In the first case $A B=A C=0, B \circ C=0$ are necessary and sufficient conditions that the line be contained in $F_{4} / P_{3}$, as by 6.7 , we need $A(s B+t C)=0$ and $(s B+t C)^{2}=0$ for all $[s, t] \in \mathbb{P}^{1}$. Moreover, $\operatorname{dim} \mathbb{G}\left(\mathbb{P}^{1}, F_{4} / P_{3}\right)=24$ because the choice of $[A]$ is 15 dimensions and then one needs an element of $G_{o}(2,8)$, which is of dimension 9 . (Here $\mathbb{C}^{8} \simeq T_{1}$.) 
In the second case, considering $F_{4} / P_{2,4}$ as a $\mathbb{P}^{2}$-bundle over $F_{4} / P_{2}$, the conditions $A B=A C=B C=0$ follow from picking an element of $F_{4} / P_{2}$, and the choice of $[A]$ is a choice of an element in the fiber.

Proof of 6.9. First, $\operatorname{dim} B=5$ because

$$
\operatorname{dim}\left(F_{4} / P_{3}\right)+\operatorname{dim} \text { Base }\left|\mathbb{F}_{F_{4} / P_{3}}^{2}\right|-1=\operatorname{dim} \mathbb{G}\left(\mathbb{P}^{1}, F_{4} / P_{3}\right) .
$$

Moreover, we know that $B$ contains $Y_{1}$ and $Y_{2}$ and is irreducible.

Consider now $\left[y_{1}+y_{2}\right] \in B$, with $y_{j} \in Y_{j} \subset \mathbb{P} T_{j}$. Write $y_{1}=e \otimes u$ and $y_{2}=e^{*} \otimes v^{2}$. Conditions for such a point to belong to $B$ can only come from components of

$$
T_{1}^{*} \otimes T_{2}^{*}=\left(E \otimes E^{*}\right) \otimes\left(U^{*} \otimes S^{2} U^{*}\right)=(\mathbb{C} \oplus \mathfrak{s l}(E)) \otimes\left(U^{*} \oplus S^{3} U^{*}\right) .
$$

Suppose that $\mathfrak{s} l(E) \otimes S^{3} U^{*}$ were contained in $N_{2}^{*}$. Since $e \otimes e^{*}$ is not a homothety, this would force $u v^{2}$ to be zero in $S^{3} U$, hence $u$ or $v$ to be zero. If this component were in $N_{2}^{*}$, then $B$ would be included in $\mathbb{P} T_{1} \sqcup \mathbb{P} T_{2}$, and would not be irreducible.

Suppose now that $\mathfrak{s l}(E) \otimes U^{*}$ were contained in $N_{2}^{*}$. This set of equations would force $u$ and $v$ to be parallel because under the contraction $U \otimes S^{2} U \rightarrow U, u \otimes v^{2}$ maps to $\omega(u, v) v$, where $\omega \in \Lambda^{2} U^{*}$. Similarly, the component $S^{3} U^{*}$ would force $\left\langle e, e^{*}\right\rangle=0$.

In conclusion, $\mathbb{P}\left\{e \otimes u \oplus e^{*} \otimes u^{2} \mid\left\langle e, e^{*}\right\rangle=0\right\} \subseteq B$. Since both sets are irreducible of dimension five, the second one must be the closure of the first one.

The quadric bundle structure is given by the application $B \rightarrow \mathbb{P} U$ defined by $\left[e^{*} \otimes u+e \otimes u^{2}\right] \mapsto[u]$. This is a nontrivial bundle structure. Finally, to see that $B$ cannot be homogeneous, note that there are no homogeneous nontrivial quadric fibrations in dimension five.

Further calculations along this line show that each variety of linear spaces is a finite union of $F_{4}$-orbits.

\section{References}

[1] N. Bourbaki, Groupes et algèbres de Lie, Hermann, Paris, 1968.

[2] E. Cartan, Sur les variétés de courbure constante d'un espace euclidien ou non-euclidien, Collected Works III, 321-432.

[3] Séminaire Chevalley (1956-1958), Classification des groupes de Lie algébriques, Paris, 1958.

[4] A. Cohen and B. Cooperstein, Lie incidence systems from projective varieties, Proc. Am. Math. Soc. 126 (1998), 2095-2102.

[5] M. Demazure, Désingularisation des variétés de Schubert généralisées, Ann. scient. Ec. Norm. Sup. 7 (1974), 53-88.

[6] J. Dixmier, Algèbres enveloppantes, Gauthier-Villars, Paris, 1974.

[7] E. B. Dynkin, Maximal subgroups of the classical groups, Amer. Math. Soc. Trans. Series 6 (1957), 245-378. 
[8] M. Green, Koszul cohomology and the geometry of projective varieties, J. Differ. Geom. 19 (1984), 125-167.

[9] P. A. Griffiths and J. Harris, Algebraic Geometry and Local Differential Geometry, Ann. scient. Ec. Norm. Sup. 12 (1979), 355-432.

[10] F. R. Harvey, Spinors and calibrations, Perspectives in Math. Vol. 9, Academic Press, 1990.

[11] B. Kostant, Lie algebra cohomology and the generalized Borel-Weil theorem, Ann. Math. 74 (1961), 329-387.

[12] J. M. Landsberg, On second fundamental forms of projective varieties, Inventiones Math. 117 (1994), 303-315.

[13] J. M. Landsberg, On degenerate secant and tangential varieties and local differential geometry, Duke Math. J. 85 (1996), 605-634.

[14] J. M. Landsberg and L. Manivel, The projective geometry of Freudenthal's magic chart, J. Algebra 239 (2001), 477-512.

[15] J. M. Landsberg and L. Manivel, Construction and classification of complex simple Lie algebras via projective geometry, Selecta Math. 8 (2002), 137-159.

[16] J. M. Landsberg and L. Manivel, Triality, exceptional Lie algebras, and Deligne dimension formulas, Advances Math. 171 (2002), 59-85.

[17] J. M. Landsberg and L. Manivel, Series of Lie groups, preprint arXiv:math.AG/0203241.

[18] J. M. Landsberg and L. Manivel, Representation theory and projective geometry, preprint arXiv:math.AG/0203260.

[19] W. Lichtenstein, A system of quadrics describing the orbit of the highest weight vector, Proc. A.M.S. 84 (1982), 605-608.

[20] LiE, A computer algebra package for Lie group computations, http://young.sp2mi.univpoitiers.fr/ ${ }^{\sim}$ marc/LiE/

[21] I. G. Macdonald, Symmetric functions and Hall polynomials, Oxford, Clarendon Press, 1979.

[22] G. C. M. Ruitenburg, Invariant ideals of polynomials algebras with multiplicity free group action, Compositio Math. 71 (1989), 181-227.

[23] T. Springer and F. Veldkamp, Octonions, Jordan algebras and exceptional groups, viii+208 pp., Springer Monographs in Mathematics, Springer-Verlag, Berlin, 2000.

[24] J. Tits, Groupes semi-simples complexes et géométrie projective, Séminaire Bourbaki 7 (1954/1955), exposé 112, 11 pages.

[25] J. Wolf, Spaces of constant curvature, Third edition, Publish or Perish 1974.

Joseph M. Landsberg

School of Mathematics

Georgia Institute of Technology

Atlanta, GA 30332-0160

USA

e-mail: jml@math.gatech.edu

\section{Laurent Manivel}

Institut Fourier, UMR 5582 du CNRS

Université Grenoble I, BP 74

38402 Saint Martin d'Hères cedex

France

e-mail: Laurent.Manivel@ujf-grenoble.fr

(Received: February 23, 2001) 